\title{
Seventh International Symposium on Intensive Care and Emergency Medicine for Latin America
}

\author{
São Paulo, Brazil. 19-22 June 2013
}

Published: 19 June 2013

\section{Basic Science}

P1

Bone morphogenetic protein-2 and leptin but not endothelin-1 induce osteochondrogenesis through increasing oxidative stress in vascular smooth muscle cells

LS do Carmo, MCC de Andrade, D de Castro Fernandes, M Liberman Albert Einstein Research and Education Institute, Morumbi, São Paulo, SP, Brazil Critical Care 2013, 17(Suppl 3):P1 (doi: 10.1186/cc12617)

Introduction Vascular calcification is a regulated process, which associates with coronary artery disease (CAD) and occurs through an increase in transcription factor expression such as RUNX2, MSX2 and alkaline phosphatase (ALP), then inducing calcium deposition. Bone morphogenetic protein-2 (BMP2) is a potent osteochondrogenic mediator, which is expressed in CAD. Endothelin-1 (ET1) and leptin have a role in regulating inflammation and CAD. We hypothesized that BMP2, leptin or both increase ROS formation in C57BL/6 vascular smooth muscle cells $(S M C)$, stimulating osteochondrogenic differentiation. We also investigated the effect of ET1 in SMC osteochondrogenesis. Our objectives were: to investigate ROS production in SMC after BMP2 $(50 \mathrm{ng} / \mathrm{ml})$ and/or leptin $(10 \mathrm{ng} / \mathrm{ml})$ incubation for 6 hours; and to assess osteochondrogenic gene expression and calcification of SMC stimulated with BMP2, leptin or ET1 (10 nM).

Methods We assessed 2-hydroxyethidium, more specific for superoxide, and ethidium which reflects hydrogen peroxide through $\mathrm{HPLC}$ analysis in SMC after stimulation. SMC cells were incubated with these stimuli for 48 to 96 hours and RUNX2, MSX2, ALP mRNA and protein expression were assessed using qPCR and western blotting. We quantified SMC calcification after 14 days of stimulation through Alizarin Red staining. Results The results are shown as mean \pm SD and were statistically significant when pHydrogen peroxide and superoxide production increased both in BMP2 and in leptin-incubated SMC (3.77 \pm 0.32 and $3.26 \pm 0.26)$ versus control $(n=6) ; \mathrm{pBMP2}$ and leptin alone increased SMC calcification $(1.25 \pm 0.08$ and $1.28 \pm 0.14)$ versus control after 14 days $(n=6)$; pET1 alone did not stimulate osteocondrogenic mRNA expression vs. control.

Conclusion We showed that BMP2 and leptin increased ROS formation in SMC, which stimulated osteocondrogenic mRNA/protein expression to induce SMC calcification. ET1 alone did not increase osteochondrogenesis in SMC.

P2

Effects of rapid repetition of a vascular occlusion test on near-infrared spectroscopy-derived variables in healthy subjects and in critically ill patients

DO Cortes, F Puflea, K Donadello, D de Backer, J-L Vincent, J Creteur Erasme Hospital, Universite Libre de Bruxelles, Anderlecht, Bruxelles, Belgium Critical Care 2013, 17(Suppl 3):P2 (doi: 10.1186/cc12618)

Introduction Transient ischemia modifies cellular metabolism and microvascular physiology in order to limit damage from future hypoxic episodes, a phenomenon called preconditioning. Near-infrared spectroscopy (NIRS) is a non-invasive technique that, when coupled to a vascular occlusion test (VOT), provides an indirect measurement of muscle oxygen consumption $\left(\mathrm{VO}_{2}\right)$ and microvascular reactivity. We hypothesized that: rapid repetition of a VOT may alter VOTinduced NIRS-derived variables and these changes could reflect preconditioning; and these alterations would be different in healthy volunteers and critically ill patients.

Methods Continuous non-invasive measurements of thenar tissue oxygen saturation $\left(\mathrm{StO}_{2}\right)$ were performed using NIRS technology (InSpectra 650; Hutchinson, USA). VOTs were performed by inflating a cuff to $50 \mathrm{mmHg}$ above the systolic pressure for 3 minutes. In a group of healthy volunteers, the VOT was repeated after 5 minutes on day 1 , after 15 minutes on day 2 and after 30 minutes on day 3 . In a group of critically ill patients, the VOT was repeated after 5 minutes. For each VOT, we calculated the $\mathrm{StO}_{2}$ desaturation slope (DescSlope), $\mathrm{StO}_{2}$ resaturation slope (AscSlope) and the $\mathrm{NIRS} \mathrm{VO}_{2}$ as the DescSlopexmean total hemoglobin index over the occlusion time. All statistical analyses were performed using SPSS 19.0 (IBM, USA).

Results Twenty-one healthy volunteers (age $29 \pm 6$ years, heart rate $71 \pm 6 \mathrm{bpm}$, mean arterial pressure $82 \pm 6 \mathrm{mmHg}$ ) and 18 critically ill patients (age $59 \pm 14$ years, APACHE II score $21 \pm 9$, norepinephrine use in 10/18, ICU mortality $22 \%$ ) were included. In the healthy volunteers, repetition of the VOT was associated with a decrease in the DescSlope and in NIRS VO. This effect was not observed in the critically ill patients (Tables 1 and 2).

Table 1 (abstract P2). Effects of a repeat VOT on VOT-induced NIRS-derived variables in healthy volunteers

\begin{tabular}{llccc}
\hline Interval & Variable & First & Second & $P$ value \\
\hline 5 minutes & AscSlope & $4.2(3.4$ to 4.9$)$ & $4(3.3$ to 5$)$ & 0.298 \\
& DescSlope & $12(9.2$ to 14.5$)$ & $9.8(8.3$ to 10.8$)$ & $>0.001$ \\
& $\mathrm{NIRSVO}_{2}$ & $151(132$ to 171$)$ & $131(118$ to 146$)$ & 0.001 \\
15 minutes & AscSlope & $4(3.2$ to 5.2$)$ & $4.1(3.4$ to 5$)$ & 0.676 \\
& DescSlope & $10.3(9.6$ to 11.3$)$ & $9.4(8.3$ to 10.2$)$ & 0.003 \\
& NIRS VO & $153(141$ to 165$)$ & $141(120$ to 146$)$ & 0.005 \\
30 minutes & AscSlope & $4.2(3.6$ to 5.3$)$ & $3.4(3.1$ to 4.8$)$ & 0.006 \\
& DescSlope & $10.9(9.5$ to 12.6$)$ & $9.4(7.4$ to 10.4$)$ & $>0.001$ \\
& $\mathrm{NIRSVO}_{2}$ & $157(122$ to 171$)$ & $132(112$ to 152$)$ & $>0.001$ \\
\hline
\end{tabular}

Table 2 (abstract P2). Effects of a repeat VOT on VOT-induced NIRS-derived variables in critically ill patients

\begin{tabular}{llccc}
\hline Interval & Variable & First & Second & $P$ value \\
\hline 5 minutes & AscSlope & $3.6(2.7$ to 4$)$ & $3.4(2.8$ to 4.6$)$ & 0.065 \\
& DescSlope & $10(8.4$ to 11.6$)$ & $10.5(8$ to 11.8$)$ & 0.774 \\
& $\mathrm{NIRSVO}_{2}$ & $103(74$ to 156$)$ & $108(73$ to 140$)$ & 0.442 \\
\hline
\end{tabular}


Conclusion Rapid repetition of a VOT alters VOT-induced NIRS-derived variables in healthy volunteers but not in critically ill patients. If these alterations reflect preconditioning, our results suggest that this phenomenon may be altered in critically ill patients.

\section{P3}

Low incidence of delirium in patients followed by physiotherapists in the ICU

KT Timenetsky, D Carnieli-Cazati, CS Azevedo, RC Eid

Hospital Israelita Albert Einstein, Morumbi, Sao Paulo, SP, Brazil

Critical Care 2013, 17(Suppl 3):P3 (doi: 10.1186/cc12619)

Introduction Delirium is an acute temporary and fluctuating mentalorganic syndrome, characterized by a global impairment of cognitive function, reduced level of consciousness, attentional deficits and altered sleep-wake cycle, and changes in arousal (hyperactive, hypoactive, or mixed). The Confusion Assessment Method (CAM and CAM ICU) [1] is a diagnostic assessment instrument for delirium and can lead physiotherapeutic treatment, aiming to optimize patient's recovery, reinforce the importance of preventive and therapeutic measures, and appraise the multidisciplinary treatment approach in this severe complicating syndrome. Delirium is present in 20 to $40 \%$ of ICU patients. So far we have no data regarding the incidence of delirium at the ICU in patients followed by physiotherapists. The objective of this study was to verify the incidence of delirium through the CAM ICU instrument in ICU patients followed by physiotherapists.

Methods Trained and capacitated physiotherapists applied the CAM ICU in patients admitted to Albert Einstein Jewish Hospital ICU, older than 18 years old and with 24 hours physiotherapy assistance per day. The content and level of consciousness investigation was performed daily in all physiotherapy sessions, which allowed characterizing the need to apply the CAM ICU. These data were evaluated twice a week during a 30-day period through medical charts.

Results During the study period, 226 patients were evaluated by physiotherapists, median age 70 years old (range 21 to 92 ), and $60 \%$ were male. The clinical admission diagnoses were: $36 \%$ sepsis, $20 \%$ cardiac, $19 \%$ neurologic, $11 \%$ respiratory, $4 \%$ orthopedic, $3 \%$ liver and gastric patients, and $2 \%$ vascular. The mean Simplified Acute Physiology Score (SAPS 3) was 45 points. We found a delirium incidence of $7 \%(n=16)$, of these patients $25 \%$ were under mechanical ventilation and $25 \%$ were spontaneously breathing. In regards to the clinical admission diagnosis, delirium was present in $20 \%$ vascular patients, $16 \%$ neurologic patients, $14 \%$ liver patients, $8 \%$ respiratory patients and $2 \%$ cardiac patients. We found no correlation of delirium with clinical admission diagnosis $(P=0.76 ; r=-0.054)$.

Conclusion There was a low incidence of delirium in ICU patients followed by physiotherapists. This may be due to physiotherapy and multi-professional team interventions performed earlier.

\section{Reference}

1. Bergeron N, Skrobik Y, Dubois MJ: Delirium in critically ill patients. Crit Care 2002, 6:181-182

P4

Relationship between clinical and cytokines profile of brain-death donors

SL Mello, M da Consolação Vieira Moreira, RMFL Silva

Faculdade de Medicina da Universidade Federa de Minas Gerais, Pampulha, Belo Horizonte, MG, Brazil

Critical Care 2013, 17(Suppl 3):P4 (doi: 10.1186/cc12620)

Introduction Brain death induces a massive inflammatory response. The majority of transplants are derived from donors who suffered from brain injury. The possible relation of clinical profile and cytokines in donors has been poorly explored. The objectives of this study were to analyze clinical characteristics of brain-dead donors and the correlation with cytokine profile in the ICU of a unique tertiary-care hospital.

Methods We evaluated 120 consecutive potential brain-dead organ donors (mean age 34.9 years, $74.2 \%$ males) between July 2007 and June 2008. Plasma cytokines (TNF, IL-2, IL-4, IL-5, IL-6, IL-8, IL-10, IFNY) were measured in 40 donors immediately after criteria for brain death (or confirmatory tests) and after obtaining consent from families. Cytokines were assessed by cytometric bead array in the plasma and all laboratory personnel were blinded to clinical information.

Results The main cause of brain death was cerebral trauma (80\%) and cerebral vascular accidents. The use of vasoactive agents was $90.6 \%$. The median time of stay in the ICU was 2 days and the mean of organs transplanted was 2.2. Data (mean pg/ml) of cytokines were: IL-2, 3.32; IL-4, 2.63; IL-5, 11.4; IL-10, 25.99; IFN, 9.72; and TNF, 2.32. In 35\% of donors IL-6 was above $5,000 \mathrm{pg} / \mathrm{ml}$ and in $15 \% \mathrm{IL}-8$ was below the detection limit of analysis. We did not find correlation (nonparametric statistical tests) between cytokines and gender, age, and laboratory tests of our organ donors. Pearson correlation between IL- 6 and TNF was 0.001 . IL-2 and IL-4, IL-5, IL-10 and IFN presented Pearson correlation $\leq 0.00$. See Table 1.

Table 1 (abstract P4). Cytokine levels

\begin{tabular}{lc}
\hline Cytokine & $P$ value \\
\hline IL-2 & 0.147 \\
IL-4 & 0.044 \\
IL-5 & 0.252 \\
IL-8 & 0.764 \\
IL-10Th2 & 0.214 \\
IFNY & 0.001 \\
\hline
\end{tabular}

Conclusion Levels of proinflammatory and anti-inflammatory cytokines were increased in brain-dead donors and were correlated. There was no difference between cytokines and clinical and laboratory profiles. References

1. Keel M, Trentz O: Pathophysiology of polytrauma. Injury 2005, 36:691-709

2. Barklin A: Systemic inflammation in the brain-dead organ donor. Acta Anaesthesiol Scand 2009, 53:425-435.

\section{Cardiology}

\section{P5}

Development of a sleep quality questionnaire to assess sleep in the ICU: a polysomnography study

LJ Storti, DM Servantes, MA Borges, FU Maroja, PR Burke, LR Bittencourt, ACC Carvalho, S Tufik, AAV de Paola, FD Cintra

Escola Paulista de Medicina - Universidade Federal de São Paulo, Vila

Clementino, São Paulo, SP, Brazil

Critical Care 2013, 17(Suppl 3):P5 (doi: 10.1186/cc12621)

Introduction Sleep is an important issue for the maintenance of cardiovascular homeostasis through heart rate and blood pressure modulation. Patients admitted in a coronary care unit (CCU) may exhibit a peculiar sleep pattern that is not fully understood. A feasible and cost-effective tool to analyze sleep in this scenario could bring important information for clinicians. The aim of this study was to evaluate sleep with a questionnaire developed specifically for the CCU and to establish correlations with polysomnography.

Methods Consecutive acute coronary syndrome patients admitted to a CCU between March 2011 and October 2012 were selected. The exclusion criteria were: hemodynamic instability, sedation, receiving vasoactive drugs, or under ventilation support. Patients were submitted to polysomnography in the first 36 hours after admission. A specific 18-question questionnaire (Storti questionnaire) divided into diurnal and nocturnal sleep was developed according to experts' skills. The Pittsburgh and the Storti questionnaires were applied immediately before the CCU discharge. Cronbach's alpha test was used for internal questionnaire validation. Spearman and Kruskal-Wallis test were used to analyze the correlation between polysomnography variables and questionnaire.

Results Ninety-nine patients (68\% male; mean age $56 \pm 10$ years) were included. The mean BMI was $27 \pm 5 \mathrm{~kg} / \mathrm{m}^{2}$. Arterial hypertension was observed in $52 \%$ of the sample; $17 \%$ had diabetes, $39 \%$ were smokers. The patients present a total sleep time of $265 \pm 81$ minutes during the polysomnography, sleep efficiency was $62 \pm 18 \%$, REM sleep was 
$10 \pm 7 \%$, and apnea/hypopnea index and arousal index was $15 \pm 23$ and $24 \pm 15$, respectively. Cronbach's test was 0.69 (range: 0.63 to 0.69 when one of the questions was removed). The Storti questionnaire also showed correlations with polysomnography $(r=0.52 ; P<0.001)$, better than the Pittsburgh questionnaire $(r=-0.25 ; P=0.02)$. When sleep profile was divided according to the questionnaire score into three categories - poor, regular and good - poor and regular sleep was observed in 64 (65\%) patients and good sleep in 35 (35\%) patients. Moreover, patients classified as good sleepers had a sleep efficiency of $72 \pm 9 \%$, better than those with a regular or poor sleep $(60 \pm 16 \%$ and $53 \pm 20 \%$, respectively; $P<0.01$ ).

Conclusion The Storti questionnaire had a good correlation with sleep efficiency assessed by polysomnography. The majority of patients in CCU had a poor or regular sleep.

P6

Serial brain natriuretic peptide strongly predicts in-hospital mortality in patients with acute myocardial infarction AE Pereira Pesaro, M Katz, C Pereira, AG Correa, AN Fava, M Franken, ACB Nunes, LM Forlenza, F Tarasoutchi, MR Makdisse

Hospital Israelita Albert Einstein, Morumbi, São Paulo, SP, Brazil

Critical Care 2013, 17(Suppl 3):P6 (doi: 10.1186/cc12622)

Introduction Brain natriuretic peptide (BNP) can be useful in risk stratification of patients with acute myocardial infarction (AMI). However, the value of serial in-hospital BNP assessment to predict mortality in this setting was poorly investigated before. Thus, the aim of this study was to evaluate the usefulness of serial BNP measurements to predict mortality in patients with AMI.

Methods Patients with AMI $(n=2,198)$ were consecutively enrolled between 2004 and 2012 in a prospective single-center registry. A subgroup analysis was performed in the patients submitted to serial BNP measurements as indicated for clinical purposes. The BNP variation was calculated based on the change between the first BNP collected (baseline) and the highest subsequent in-hospital BNP measurement. Baseline characteristics and the BNP variation were compared between patients who survived or died during the in-hospital period. Categorical variables were compared by Pearson's chi-square test. Continuous variables were compared using the Student $t$ test or Mann-Whitney test. The logistic regression model was used to test the association between BNP increase (categorized by an increase $\geq 200 \mathrm{pg} / \mathrm{ml}$ ) and mortality, adjusted for age, gender, troponin levels, Killip classification, left ventricular ejection fraction (LVEF) and ST elevation AMI. $P<0.05$ was considered statistically significant.

Results Serial BNP levels were determined in 280 patients (59\% men, $78 \pm 12$ years, 33\% ST elevation). The BNP increase $>200 \mathrm{pg} / \mathrm{ml}$ was detected in 114 (41\%) patients. All baseline clinical parameters (gender, age, diabetes, hypertension, Killip classification, LVEF and ST elevation) were similar between patients with or without BNP increase. Mortality was higher in patients with BNP increase ( $25 \%$ vs. $12 \% ; P=0.006)$. In the adjusted logistic regression model, only age $(\mathrm{OR}=1.04 ; 95 \% \mathrm{Cl}=1.002$ to $1.08 ; P=0.04)$ and BNP increase $>200 \mathrm{pg} / \mathrm{ml}(\mathrm{OR}=3.9 ; 95 \% \mathrm{Cl}=1.83$ to $8.20 ; P<0.001$ ) were independent predictors of in-hospital mortality. Conclusion This study demonstrated that an in-hospital BNP increase $>200 \mathrm{pg} / \mathrm{ml}$ is strongly and independently related to in-hospital mortality in patients with AMI. Thus, serial BNP testing may be useful to detect high-risk AMI patients.

\section{Epidemiology/Quality of Life/Administration}

\section{P7}

Causes of ICU readmission and mortality: analysis of a 6-month period

PG Brandão, J Syrio, L Machado, M Guimarães, MV Lopes, SA Lobo Hospital de Base de São José do Rio Preto, São Manoel, São Jose do Rio Preto, SP, Brazil

Critical Care 2013, 17(Suppl 3):P7 (doi: 10.1186/cc12623)

Introduction Patients readmitted to ICU have a higher mortality and longer ICU and hospital stay. Furthermore, the readmission rate is used as a quality indicator of critical care unit performance, because this index may reflect the adequacy of treatment. The objective was to evaluate the readmission rate of a tertiary public hospital during a 6-month period.

Methods We performed a retrospective analysis of all adult patients readmitted to a 20-bed mixed-case ICU between 1 September 2012 and 28 February 2013. The cases (readmission) were collected from clinical electronic information system.

Results During this period 402 patients were admitted to the ICU. The mortality in the ICU was $24.6 \%$ and overall hospital mortality rate was $31.6 \%$. The average SAPS 3 during readmission on the ICU was 52 with a predicted mortality of $34 \%$. The readmission rate was $5.2 \%$, ICU mortality was $23.8 \%$ and hospital mortality was $28.6 \%$. The most frequent cause of readmission was nosocomial pneumonia (29\%), neurologic causes (19\%), sepsis (14\%), administrative (14\%), postoperative support (10\%), metabolic disorders (10\%) and cardiology events (5\%). The patients were most commonly readmitted form the ward (33\%), emergency department (14\%), step-down unit (14\%), operating theater (5\%) and others (33\%). The most common supportive therapies after readmission were mechanical ventilation in $38.1 \%$, vasopressors in $28.6 \%$, and renal support in $9.5 \%$.

Conclusion The most common reason for ICU readmission in our unit was nosocomial pneumonia. The mortality of the readmitted patients was not superior to the predicted mortality for the overall cohort of patients.

P8

Cost of the quantitative adequacy of nursing staff in the ICU

CP Guimarães', PC Garcia², FMT Fugulin

'Escola de Enfermagem da Universidade de São Paulo, SP, Brazil; ${ }^{2}$ Hospital

Universitário da Universidade de São Paulo, SP, Brazil

Critical Care 2013, 17(Suppl 3):P8 (doi: 10.1186/cc12624)

Introduction The high cost to maintain a complex structure such as the ICU has justified its strict control. Nevertheless, budgetary limitation and expenses abatement directly affect the outcome of a fully adequate nursing staff due to its high-percentage representation in human resources. Therefore, the lack of proper personnel interferes on many aspects of daily activities such as organization and safety of patients and staff, affecting also the assistance provided and the institutional goals. For that reason, study on costs and management of nursing personnel is paramount, as it can evidence the effects of an impaired scenario and the relation between cost and efficiency in a healthcare environment. The objectives were: to verify the average staff time required by a patient for a proper assistance or treatment; to calculate the actual average time and cost spent by the crew; and to estimate the average daily time and cost for a proper adult ICU's activity.

Methods The research was based on a quantitative and descriptive data. The study took place in the Hospital Universitário da Universidade de São Paulo's adult ICU block, from 1 January 2008 to 31 December 2009. Data concerning the average time of assistance given to the patients and requested by them were collected from the ICU's management instruments. Data concerning personnel fees per hour were based on the ICU nursing staff's wage bill, provided by the Finance Department. Results The daily average time of required assistance is 16 hours. However, the actual daily average time of provided assistance is 14 hours, which poses a great disparity statistically. In 24 hours the average cost of given assistance per patient was $\mathrm{R} \$ 715.79$. On the other hand, adequate assistance would require $\mathrm{R} \$ 805.66$. The average cost per month to amend the actual scenario would be $R \$ 40,490.00$, which corresponds to an increase of $17.16 \%$ over the existing outline's budget.

Conclusion The literature review and the data suggest that although the adequacy of the nursing staff entails higher costs, it may contribute to improve the quality of care, reducing costs arising from possible negative outcomes in patients.

References

1. Bochembusio L, Fugulin MTF, Lima AFC, et al.: Custo da adequação quantitativa de profissionais de enfermagem em Unidade Neonatal. Rev Escola Enferm USP 2011, 45:1582-1588.

2. Garcia PC: Tempo de assistência de Enfermagem em UTI e indicadores de qualidade assistencial: análise correlacional. Dissertação. São Paulo: Escola de Enfermagem, Universidade de São Paulo; 2011. 
3. Fugulin FMT, Gaidzinski RR: Dimensionamento de pessoal de enfermagem em unidade de terapia intensiva. In Programa de Atualização em Enfermagem: Saúde do Adulto (PROENF) - Ciclo 3 - Módulo 3. Porto Alegre: Artmed/Panamericana; 2008:65-96

P9

Factors associated with prolonged ICU stay: a retrospective analysis FG Zampieri, F Colombari, C de Batista Lovatto Pastore, C Santoro, D Haib, JP Ladeira

Hospital Alemão Oswaldo Cruz, Paraíso, São Paulo, SP, Brazil

Critical Care 2013, 17(Suppl 3):P9 (doi: 10.1186/cc12625)

Introduction Critically ill patients frequently stay on the ICU for prolonged periods. Prolonged ICU stay (PIS) is associated with increased costs, resource use and family burden. Nevertheless, risk factors at admission associated with prolonged ICU stay are only partially described. The objective was to evaluate factors associated with prolonged ICU stay on a mixed ICU.

Methods Retrospective analysis of 3,257 patients admitted to a tertiary hospital in São Paulo, Brazil. Twenty-seven relevant variables that were clinically associated with prolonged ( $\geq 14$ days) were included on a univariate analysis. Variables included demographic data, reason for admission, type of admission (clinical, elective surgery, emergency surgery), previous status performance, presence of comorbidities, illness severity (assessed by SAPS 3 score), laboratorial data and need for organ support device (vasopressors, mechanical ventilation, dialysis) on the day of admission. A multivariate analysis was performed to identify variables independently associated with PIS.

Results In total, 203 (6.3\%) of the 3,257 patients admitted in the analyzed period stayed on the ICU for at least 14 days. Hospital mortality was higher in patients with PIS $(49.7 \%$ vs. 9.8\%; $P<0.01)$. On multivariate analysis, SAPS $3(\mathrm{OR}=1.03, \mathrm{Cl}=1.01$ to 1.04$)$, reduced status performance (dependency for one or more daily activities $\mathrm{OR}=1.71, \mathrm{Cl}=1.18$ to 2.46$)$, bedridden status $(\mathrm{OR}=1.91, \mathrm{Cl}=1.08$ to 3.38), emergency surgery $(\mathrm{OR}=2.87, \mathrm{Cl}=1.27$ to 6.51$)$, admission due to intracranial mass effect $(\mathrm{OR}=4.46, \mathrm{Cl}=1.16$ to 17.04$)$, admission from the ward $(\mathrm{OR}=3.35, \mathrm{Cl}=1.05$ to 10.63) and hospital transfer (OR = $5.23, \mathrm{Cl}=1.62$ to 16.91 ) were independently associated with PIS. Age was not related to PIS. No comorbidity or organ support device was independently associated with PIS.

Conclusion In a large database of critically ill patients, global illness severity, baseline status performance and emergency surgery were related to PIS. No comorbidity or need for organ support device was associated with PIS.

Acknowledgement The authors would like to thank Dr Marcelo Park for helping with statistical analysis.

P10

Feasibility of transitioning from APACHE II to SAPS III as prognostic model in a Brazilian general ICU

A Serpa Neto, MSC Cesar de Assunção, A Pardini, NS Pinheiro de Goes, E Silva Hospital Israelita Albert Einstein, Morumbi, São Paulo, SP, Brazil

Critical Care 2013, 17(Suppl 3):P10 (doi: 10.1186/cc12626)

Introduction Prognostic models reflect the population characteristics of the countries which they originate from. The development of the Acute Physiology and Chronic Health Evaluation II (APACHE II) system was based on a cohort of patients in the United States, and it has been used in many ICUs around the world. Newer score systems were developed as the Simplified Acute Physiology Score III (SAPS III) that was developed and validated in a multicenter and multinational cohort study. Predictive models should be customized to fit in the case-mix where they will be used because the outcomes in the original databases and period from which the models were derived may be different from the databases of ICUs using the models. In the present study, we performed the external validation of two predictive models and directly compared their performance in an independent population of mixed critically ill patients in Brazil. The aim is to assess the feasibility of transitioning from APACHE II to SAPS III.

Methods Data were retrospectively collected only for APACHE II during August 2011 and December 2011, and only for SAPS III during May
2012 and September 2012. From January 2012 to April 2012, during a period of calibration, the two scores were calculated in all patients admitted to the ICU and were collected for analysis. All ICU admissions were enrolled during the period analyzed. The exclusion criteria were: age $<18$ years, missing data, and not receiving ICU care. The calibration of the scores was tested using the Hosmer-Lemeshow goodness-offit procedure. The discriminative ability of the models was assessed using receiver operating characteristic (ROC) curves and respective areas under curves (AUC). The standardized mortality ratio (SMR) was calculated using the models by dividing the number of observed deaths by the number of expected deaths. Confidence intervals of the SMR were computed to test the model's uniformity-of-fit and were calculated using the proposed methods.

Results A total of 3,333 ICU admissions were enrolled until the end of September 2012. The Hosmer-Lemeshow goodness-of-fit statistics supported model fit of all models for in-ICU mortality with the exception of APACHE II in patients in the calibration database undergoing elective surgery. For in-hospital mortality there is a worse fit of APACHE II in clinical patients during the first period and of SAPS III in patients in the calibration database undergoing elective surgery. The calibration curves for APACHE II and SAPS III shows overestimation of the risk of death in all ranges of predicted mortality. Discrimination, as tested by the AUC, in general and clinical patients was best for SAPS III for in-ICU and in-hospital mortality. SMRs for the whole population were $0.27(\mathrm{Cl}=0.23$ to 0.33$)$ for APACHE II and $0.28(\mathrm{Cl}=0.22$ to 0.36$)$ for SAPS III. In the calibration database, the SMRs for APACHE II and SAPS III were $0.33(\mathrm{Cl}=0.22$-to 0.50$)$ and $0.36(\mathrm{Cl}=0.25$ to 0.55$)$, respectively. For all models, the SMRs showed some variation across the spectrum of patients. The SMRs ranged from 0.24 to 0.46 for APACHE II, and 0.09 to 0.31 for SAPS III. In the calibration database, the SMRs ranged from 0.13 to 0.38 for APACHE II, and from 0.18 to 0.40 for SAPS III.

Conclusion The external validation of two widely used prognostic models showed good discrimination and good calibration when applied to the same independent population of Brazilian ICU patients. The transition from APACHE II to SAPS III in this Brazilian ICU was feasible and in some scenarios the SAPS III had even better performance than APACHE II. In conclusion, we showed in a cohort of Brazilian patients from a tertiary hospital that SAPS III is the best prognostic score, with the highest discrimination and calibration power. The transition from an older score (APACHE II) to a newer one (SAPS III) is feasible in this scenario.

P11

Improving quality indicators for the treatment of acute myocardial infarction: impact of the disease-specific care certification

PG Melo de Barros e Silva, MY Okada, S Simoes, VA Fernandes, TA Macedo, DL Ramos, MJ Rodrigues, MC do Amaral Baruzzi, V Furlan

Hospital Totalcor, Cerqueira César, São Paulo, SP, Brazil

Critical Care 2013, 17(Suppl 3):P11 (doi: 10.1186/cc12627)

Introduction Registries have shown that there still exists a large gap between what is recommended by evidence-based guidelines and what is actually offered to the patient in clinical practice. Monitoring quality indicators allows the identification of these gaps and, in consequence, enables specific interventions for improvement. In January 2012 began, in a Brazilian private hospital, the implementation of the Clinical Care Program (CCP) for acute myocardial infarction (AMI). The present study aims to evaluate the impact of the CCP in quality indicators for AMI including in-hospital mortality.

Methods All patients with a confirmed diagnosis of AMI (with or without ST elevation), after signing consent, become part of the CCP, and all care would be managed by a dedicated nurse who mobilizes a multidisciplinary team, checking records, organizing and monitoring indicators. Four indicators that are part of the Joint Commission International Library of measures were monitored before and after the implementation of the program: administration of acetylsalicylic acid (ASA) in the first 24 hours; prescription of ASA, $\beta$-blockers and ACEI/ ARB at discharge, excluding patients with contraindications. In-hospital mortality was also assessed. A comparison of the year before (Group I - 2011) versus the year after (Group II - 2012) initiation of the CCP was made. Statistical analysis included the calculation of point estimates 
and $95 \%$ Cls. Comparison of categorical variables was performed by chi-square and a two-tailed significant $P$ value.

Results During 2011 and 2012, a total of 776 patients had the diagnosis of AMI in our service (Table 1).

Table 1 (abstract P11). Comparison between Groups I and II

\begin{tabular}{lccc}
\hline AMI patients & $\begin{array}{c}\text { Group I - 2011 } \\
(\boldsymbol{n}=\mathbf{3 5 2})\end{array}$ & $\begin{array}{c}\text { Group II - 2012 } \\
(\boldsymbol{n}=\mathbf{4 2 4})\end{array}$ & P value \\
\hline ASA in the first 24 hours & $96 \%(94$ to $98 \%)$ & $100 \%(99$ to $100 \%)$ & $<0.001$ \\
ASA at discharge & $99 \%(97$ to $100 \%)$ & $100 \%(99$ to $100 \%)$ & NS \\
B-blocker at discharge & $94 \%(92$ to $96 \%)$ & $99 \%(98$ to $100 \%)$ & 0.001 \\
ACEI/ARB at discharge & $95 \%(93$ to $97 \%)$ & $99 \%(98$ to $100 \%)$ & 0.001 \\
In-hospital mortality & $5.1 \%(3.2$ to $8 \%)$ & $2.6 \%(1.4$ to $4.6 \%)$ & 0.06
\end{tabular}

$95 \% \mathrm{Cl}$ in parentheses. $\mathrm{ACEl}$, angiotensin-converting enzyme inhibitor; $\mathrm{ARB}$, angiotensin receptor blockers.

Conclusion After beginning the CCP for AMI, a significant improvement in quality indicators occurred. Reduction of hospital mortality had a $P$ value near the threshold for statistical significance.

Acknowledgements This project is funded by Hospital Totalcor and Amil Clinical Research.

\section{P12}

Laboratory routine in the ICU: a practice to be abolished?

AA Peixoto $\mathrm{Jr}^{1,2}$, FA Meneses ${ }^{1,3}$, BP Barbosa', LFP Pessoa', RHT Melo',

GM Fideles ${ }^{2}$

'Centro Universitário Christus, Papicu, Fortaleza, CE, Brazil; ${ }^{2}$ Hospital

Universitário Walter Cantídio - UFC, Papicu, Fortaleza, CE, Brazil; ${ }^{3}$ Hospital

Geral de Fortaleza - SESA, Papicu, Fortaleza, CE, Brazil

Critical Care 2013, 17(Suppl 3):P12 (doi: 10.1186/cc12628)

Introduction The execution of laboratory tests is frequently requested for diagnosis and/or monitoring of critical patients. Their role as an aid, however, is diminished by unreasonable practices - resulting, unfortunately, in iatrogenesis and higher costs. In face of these ominous results, we tracked the laboratory tests performed routinely in an ICU. Methods We retrospectively analyzed the results of tests performed on a daily and consecutive basis during the months of May and June 2012. We deliberately restricted our analysis to the levels of creatinine, urea, sodium, and potassium, and to the prothrombin time (PT) and activated partial thromboplastin time (aPTT) tests. To infer their propriety, we compared each result with their respective normality references. In addition to comparing the cumulative rates of normal tests, we were also interested in the correlation of the results and the admission APACHE II score and time of hospitalization in the ICU, as well as in the volumes of blood drawn and in the test costs.

Results Forty-eight patients ( 28 men) were studied, with a mean age of $46.6 \pm 18.6$ years, average APACHE II score of $16.5 \pm 6.9$ points, average length of stay in the ICU of $15.2 \pm 11.7$ days, and $33 \%$ fatality rate. $A$ total of 3,622 tests were performed (90.6/deceased patient $\times 67.1 /$ surviving patient), with a predominance of potassium (13.5\%), sodium (13.3\%), and creatinine (13.3\%) levels, and complete blood count, $(13.2 \%)$. We observed a linear correlation $(r=0.81 ; P<0.05)$ between the number of performed tests/patient and the respective length of stay in the ICU; no correlation was observed for the APACHE II score. The volume of blood drawn per patient per hospitalization varied between 10 and $525 \mathrm{ml}$, being higher for deceased patients (average of $103.5 \times 84.2$ ). Of the total tests, $48.8 \%$ of the results were normal (43.9 tests/surviving patient $\times 44.9$ tests/deceased patient), especially for potassium levels $(9.8 \%)$ and aPTT $(9.2 \%)$. Also, $31.8 \%$ of all results were consecutively normal, specially potassium levels $(7.1 \%)$ and aPTT (6.8\%). A total of approximately US $\$ 65,000$ was spent in tests for which results were normal.

Conclusion The collected data reveal that almost one-half of the tests resulted in normal values, with the aggravating factor of consecutivity. This authorizes us to question the routine practice of laboratory tests, highlighting their possible meaninglessness for decision-making, as well as their implication in unreasonable blood spoliation (culminating in transfusions) and their respective costs (approximately US\$4,000 annually).

P13

Nursing care time and quality indicators for the ICU: correlation analysis

PC Garcia', FMT Fugulin²

'Hospital Universitário da Universidade de São Paulo, Cidade Universitária, São Paulo, SP, Brazil; '2Escola de Enfermagem, Universidade de São Paulo, SP, Brazil

Critical Care 2013, 17(Suppl 3):P13 (doi: 10.1186/cc12629)

Introduction The objective of this exploratory, retrospective, quantitative study was to analyze the time spent by the nursing staff to assist patients in an adult ICU (AICU) of the University Hospital, University of São Paulo (UH-USP) and verify its correlation with quality care indicators.

Methods This research started on 1 January 2008 until 31 December 2009. Data were collected from the administration tools used by the head nursing staff of the unit. Analysis of the average length of time in relation to the average length of time required by patients was performed using the paired $t$ test. The correlation coefficient was used to verify the correlation of the average length of care time given to patients in the AICU with the quality indicator incidence.

Results The average length of time regarding assistance for patients, in the analyzed period, accounted for 14 hours, of which $31 \%$ were performed by nurses and $69 \%$ by technicians/nursing assistants. The hospitalized patients required approximately 16 hours of care. The application of statistical tests showed that the differences found between the hours of assistance given by the nursing staff and those required by patients was significant $(P<0.001)$, suggestive of the heavy workload for the nurses in the AICU. The correlational analysis between the length of time of nursing care given by nurses and the quality indicator incidence of accidental extubation evidences Pearson's correlation coefficient $(r=-0.454)$, with $P=0.026$, indicating negative linearity between variables, which allowed us to infer that the incidence of accidental extubation decreases with increasing nursing care time given by nurses.

Conclusion The results revealed that the average hours of nursing care for patients of the AICU were lower than those recommended by the official Brazilian Agencies. The average time of care required by patients hospitalized in this unit was higher than that recommended by ANVISA and lower than that established by COFEN Resolution Nr. $293 / 04$. This indicates that the quantitative assessment of nursing staff in ICUs requires prior knowledge of the users' healthcare demands and not only the use of parameters indicated by official agencies, since this procedure may cause an overdimensioning or underdimensioning of nursing professionals. The results of this study showed the influence of nursing care time provided by nurses in the outcome of care given to patients assisted in the AICU. The addition of more evidence may help to demonstrate the impact of nursing care time in healthcare results and patient safety.

\section{P14}

SAPS 3 as a predictor admission of surgical patients in the ICU

AMR Rosa de Oliveira, JM Silva Jr, H Rocha, LM Sá Malbouisson,

MJC Carmona

Hospital das Clínicas da Faculdade de Medicina da Universidade de São

Paulo, Cerqueira Cesar, São Paulo, SP, Brazil

Critical Care 2013, 17(Suppl 3):P14 (doi: 10.1186/cc12630)

Introduction Owing to lack of intensive care beds, patients undergoing intermediate-risk surgery are usually sent to the ward postoperatively. However, a part from this population evolves with complications requiring intensive care (ICU). The aim of the study was to evaluate the characteristics of surgical patients who were admitted to the ICU lately and to find predictors of the need for intensive care.

Methods A prospective cohort study was performed in a tertiary hospital for 1 year. The study included patients with preoperative indication for ICU, but who at the end of surgery were taken to the 
ward postoperatively because of good clinical surgery. We evaluated the need for the ICU in this group, the SAPS 3 score preoperatively, the ASA physical status, demographics, origin and service requestor, need for blood transfusions intraoperatively, surgery time and hospital mortality. Patients undergoing palliative surgery were excluded. Independent predictors of the need for intensive care were assessed using logistic regression, with the sensitivity and specificity studied by ROC curve. See Figure 1.

Results We included 100 patients aged $66.4 \pm 14.7$ years. The SAPS 3 score average was $38.5 \pm 8.6,71 \%$ had ASA 2, women constituted $66 \%$ of casuistic. Most surgery was elective, the most frequent gynecologic (30\%) and orthopedic (28\%) and neuraxial regional anesthesia (49\%). Of all patients, $27 \%$ required ICU admission, average on the sixth day after surgery and $3.0 \%$ died. The SAPS 3 score average was higher $(45.4 \pm 7.8$ vs. $35.9 \pm 7.4, P<0.001)$ and ASA 3 was more prevalent $(40.7 \%$ vs. $8.2 \%$, $P=0.001)$ in patients who required intensive care postoperatively. Furthermore, these patients had longer duration of surgery $(4.2 \pm 1.9$ vs. $2.7 \pm 1.5$ hours, $P<0.001$ ), higher prevalence of gastrointestinal surgery $(14.8 \%$ vs. $5.5 \%, P=0.03)$ and greater need for intraoperative transfusion $(18.5 \%$ vs. $5.5 \%, P=0.04)$. In these patients admitted to the ICU mortality was $11.1 \%$ versus $0.0 \%, P=0.004$. In multivariate analysis, we found the value of SAPS 3 as an independent factor in determining whether the patient would need the ICU, OR $=1.25 ; 95 \% \mathrm{Cl}=1.1$ to 1.4 ;

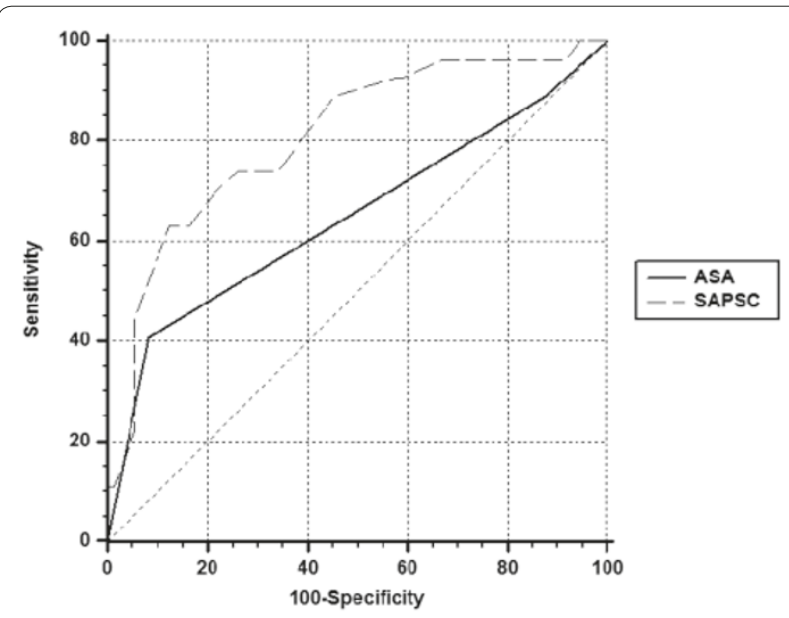

Figure 1 (abstract P14).
$P=0.001$, and even the time of surgery, $\mathrm{OR}=3.33 ; 95 \% \mathrm{Cl}=1.7$ to 6.3 ; $P=0.002$. The ROC curve was $0.87 ; 95 \% \mathrm{Cl}=0.78$ to 0.93 for the SAPS 3 discriminating need for intensive care, rather than ASA, ROC 0.64; $95 \%$ $\mathrm{Cl}=0.54$ to 0.74 .

Conclusion The identification of high-risk surgical patients is a difficult task, but essential for their proper treatment, surgery time together with the SAPS 3 seem to be useful tools in this differentiation and may help to better characterize this population.

\section{P15}

Severity-adjusted resource use and outcomes of an ICU of a tertiary hospital in Sao Paulo, Brazil

EGL Guadalupe', E Silva', F Colombari'1,2, A Serpa Neto', A Pardini'

${ }^{1}$ Hospital Israelita Albert Einstein, Morumbi, São Paulo, SP, Brazil;' ${ }^{2}$ Hospital Alemão Oswaldo Cruz, Paraíso, São Paulo, SP, Brazil

Critical Care 2013, 17(Suppl 3):P15 (doi: 10.1186/cc12631)

Introduction Quality management in intensive care currently emphasizes outcome linked to optimization of resources. Intensive care medicine focuses on the most severe patients in the hospital and therefore high resource consumption, making its management a challenge. Of many parameters to estimate utilization of resources, only length of stay (LOS) is systematically collected. Therefore, severityadjusted resource use (SRU) can be an important tool for estimating resource consumption in an ICU. The objective of this study was to evaluate the resource consumption by SRU in the adult ICU of the Hospital Israelita Albert Einstein, São Paulo, Brazil, in patients admitted during the first half of 2012.

Methods Retrospective analysis of 1,441 patients admitted during the first half of 2012 to a tertiary hospital in São Paulo, Brazil. Patients were divided into nine categories based on SAPS 3 as shown in Table 1, and then the standardized mortality rate (SMR), the quotient of observed to predicted mortality, was calculated. SRU was calculated for each stratum of SAPS 3 by dividing the LOS in the ICU for all patients by the number of survivors. The ICUs' SAPS 3 database was considered to standardize SRU. The amount of resources used was measured as the number of days per survival. Readmitted patients over 24 hours were excluded.

Results A total of 1,441 patients were analyzed. The male proportion, age, LOS and SAPS 3 averages were $57.4 \%, 65 \pm 18$ years, $3.87 \pm 5.95$ days and $45.14 \pm 15.9$, respectively. The main results are shown in Table 2. No patient died of category 2.

Conclusion According to these data, the analyzed ICU appears to be very efficient for outcomes standardized by SAPS 3 and utilization of resources by SRU. However, confounding factors should be considered: decalibration SAPS 3 for this ICU and the presence of a step-down unit in the hospital.

Table 1 (abstract P15). SAPS 3 based on categories of severity

\begin{tabular}{lccccccccc}
\hline Category & $\mathbf{1}$ & $\mathbf{2}$ & $\mathbf{3}$ & $\mathbf{4}$ & $\mathbf{5}$ & $\mathbf{6}$ & $\mathbf{7}$ & $\mathbf{8}$ & $\mathbf{9}$ \\
\hline SAPS 3 points & 0 to 24 & 25 to 34 & 35 to 44 & 45 to 54 & 55 to 64 & 65 to 74 & 75 to 84 & 85 to 94 & $>95$ \\
\hline
\end{tabular}

Table 2 (abstract P15). Data with SAPS 3, SRU and SMR

\begin{tabular}{|c|c|c|c|c|c|c|c|}
\hline Category & $\begin{array}{c}\text { SAPS 3, } \\
\text { mean (SD) }\end{array}$ & Patients & Survivors & $\begin{array}{l}\Sigma \text { days LOS } \\
\text { (all patients) }\end{array}$ & SRU & $\begin{array}{c}\text { SRU } \\
\text { standardized }\end{array}$ & SMR \\
\hline 1 & $19.75 \pm 4.08$ & 112 & 111 & 161 & 1.45 & 0.03 & 1.1 \\
\hline 2 & $30.36 \pm 2.92$ & 335 & 335 & 673 & 2.01 & 0.13 & 0 \\
\hline 3 & $39.31 \pm 2.77$ & 350 & 346 & 823 & 2.37 & 0.16 & 0.13 \\
\hline 4 & $49.18 \pm 2.86$ & 304 & 295 & 1,087 & 3.68 & 0.22 & 0.13 \\
\hline 5 & $59.06 \pm 2.79$ & 202 & 174 & 1,155 & 6.64 & 0.23 & 0.3 \\
\hline 6 & $68.77 \pm 2.79$ & 86 & 73 & 795 & 10.89 & 0.16 & 0.22 \\
\hline 7 & $78.5 \pm 2.45$ & 34 & 18 & 251 & 13.94 & 0.05 & 0.56 \\
\hline 8 & $88.54 \pm 2.99$ & 13 & 7 & 86 & 12.28 & 0.02 & 0.5 \\
\hline 9 & $97.8 \pm 4.21$ & 5 & 2 & 16 & 8 & 0.003 & 0.62 \\
\hline Total & $45.14 \pm 15.9$ & 1,441 & 1,361 & 5,047 & 3.72 & 0.13 & 0.24 \\
\hline
\end{tabular}




\section{References}

1. Rothen HU, Stricker K, Einfalt J, et al:: Variability in outcome and resource use in intensive care units. Intensive Care Med 2007, 33:1329-1336.

2. Moreno RP, Metnitz PGH, Almeida E, et al:: SAPS 3 - from evaluation of the patient to evaluation of the intensive care unit. Development of a prognostic model for hospital mortality at ICU admission. Intensive Care Med 2005, 31:1336-1355.

P16

Use of drugs in the ICU and its iatrogenic potential

É de Castro Vieira, NM Vidal, GMA Fideles, EC Santos, AA Peixoto Jr,

FA Meneses

Hospital Geral de Fortaleza, Papicu, Fortaleza, CE, Brazil

Critical Care 2013, 17(Suppl 3):P16 (doi: 10.1186/cc12632)

Introduction Developed and administered for the purpose of benefit pharmaceutical agents can cause harmful effects to the patient. We analyzed their use in a population of patients admitted to the ICU, trying to detect their potential exposure to drug-drug interactions.

Methods Prospective study of patients hospitalized for more than 48 hours, during the period from May to July 2012, with tracking of drug interactions according to Micromedex (Version 2.0).

Results We analyzed 50 patients with a mean age $45.4 \pm 18.7$ years, the majority were female (54\%) and from the emergency unit (74\%). The mean APACHE II score was $17.6 \pm 7.3$ points, the mean SOFA score (day 1) $7.3 \pm 4.2$ points, and the median length of stay 21 (IQR: 12.5 to 31.5 ) days. One hundred and three drugs were prescribed, predominantly antimicrobials (100\% of patients) and analgesics ( $98 \%$ of patients). The average/day/patient of prescription drugs was $10 \pm 2.6$, and the average/day/patient drug interactions $2.7 \pm 2.8$. On exposure to drug interactions important and moderate risk occurred, respectively, in $78 \%$ and $86 \%$, identifying the association contraindicated in $34 \%$ of patients of a positive correlation between length of stay in the ICU and exposure to important risk interactions $(r=0.53, P=0.00006)$ and moderate risk interactions $(r=0.34, P=0.013)$. Patients exposed to important risk interactions had greater severity at ICU admission for the APACHE II (18.5 \pm 7.1 vs. $14.3 \pm 6.9$ points, $P=0.045)$ and SOFA (day 1) $(7.8 \pm 4$ vs. $4.2 \pm 3.9$ points, $P=0.017)$.

Conclusion The high numbers of drug interactions with important risk incidents, especially in the sick population, alert us to the necessity of knowledge by the intensivist for the use of drugs, due to the iatrogenic potential exacerbating the severity already underway.

\section{References}

1. Hammes JA, et al:: Prevalence of potential drug-drug interactions in intensive care units. Brazilian J Intensive Care 2008, 20:349-354.

2. Rossignoli PdS, Guarido CF, Cestari IM: Occurrence of drug interactions in the intensive care unit: evaluation of medical prescriptions. Brazilian J Pharmacol 2006, 87:104-107.

\section{Hemodynamics/Shock}

\section{P17}

Analysis of the efficacy of an experimental expert system of medical prescription in reducing medical errors and excessive physician workload: a cross-sectional study

HH Shieh, ER Barreira, EJ Troster, SC Brassica, AC Ventura, PF Góes,

I de COF Fernandes, DC de Souza, JC Fernandes, F Pereira das Chagas, R de Jesus, LO Zagne, FR Caino, AE Gilio, G Galvão de França, M Luglio,

A Bousso

University Hospital of Universidade de São Paulo, Hospital Cruz Azul de São

Paulo, Instituto da Criança do HC-FMUSP, Hospital Israelita Albert Einstein,

Vila lara, São Paulo, SP, Brazil

Critical Care 2013, 17(Suppl 3):P17 (doi: 10.1186/cc12633)

Introduction Deaths attributable to preventable medical errors (PME) in hospitals exceed those caused by well-known life-threatening conditions, such as motor vehicle accidents, breast cancer, and AIDS [1]. The Institute of Medicine estimates that as many as 98,000 deaths are caused by PME every year [1]. The risks derived from PME are even more severe when they affect critically ill patients, or include medications that must be adjusted for the patient's body weight. Fatigue and work overload can represent a threaten to the patients' safety in pediatric ICUs [2]. Expert Systems (ES) [3], a branch of artificial intelligence, can be used to solve the problems related to medical prescription errors (MPE). Studies analyzing the role of ES in MPE are still lacking. The objective of this study was to compare the accuracy of an experimental ES with the written medical prescription.

Methods After signing an informed consent, pediatricians working in a university hospital were asked to write a medical prescription containing 10 different medications (maintenance fluids, adenosine, epinephrine, atropine, phenytoine, vancomycin, ceftazidime, amphothericin B, dobutamine, and fentanyl) for a hypothetical patient. The written medical prescription was compared with the ES prescription made by the same physicians, after a 2-minute training period. Statistical analysis was done using the $X^{2}$, Fisher's exact test, paired $t$ test or Wilcoxon test (paired samples), whenever applicable. A significance level of 0.05 was used for all analyses.

Results Thirteen pediatric residents and seven attending physicians participated on the study; the mean time since medical graduation was $10.1 \pm 9$ years. Fifty-seven prescribing errors were detected on medical prescription (nine unreadable items, 23 omissions, six dosing errors, 14 dilution errors and five velocity of infusion errors) in comparison with one error of duplication of medication in the ES prescription $(P<0.001)$. Conclusion The medical prescription of critically ill pediatric patients deserves special attention. The use of an experimental ES required a short training period and resulted in a significant decrease in prescribing errors and physicians' workload. Nevertheless, this computerized approach is not error free, and double-checking must be performed by the prescriber physician.

References

1. To Err is Human: Building a Safer Health System. Institute of Medicine; 1999.

2. Montgomery, Vicki L: Effect of fatigue, workload, and environment on patient safety in the pediatric intensive care unit. Pediatr Crit Care Med 2007, 8:S11-S16.

3. Shortliffe EH: Medical expert systems - knowledge tools for physicians. West J Med 1986, 145:830-839.

P18

Update of the pediatric hypertension graphic adjusted for gender and height percentiles: systolic blood pressure for boys, 1 to 17 years old

HH Shieh, AE Gilio, VHK Koch, DC Raulik, CVranjac, S Fukugava, ER Barreira, EJ Troster

University Hospital of Universidade de São Paulo, Vila lara, São Paulo, SP, Brazil Critical Care 2013, 17(Suppl 3):P18 (doi: 10.1186/cc12634)

Introduction Hypertension is the most important preventable risk factor for premature death worldwide. It increases the risk of ischemic heart disease, strokes, peripheral vascular disease, and other cardiovascular diseases, including heart failure, aortic aneurysms, diffuse atherosclerosis, and pulmonary embolism. In childhood, hypertension can be determined according to a table adjusted for height, age and gender [1]. A graphic representation of pediatric hypertension was published in 1987 [2], and no graphic updates have been published since then. The objective of this study was to update the graphic representation of pediatric hypertension.

Methods We used a computerized calculation method to develop high-resolution graphics containing curves with 5,841 points each, to depict the main percentiles associated with high blood pressure for boys from 1 to 17 years old in the 50th percentile of height. Each point represents the calculation of the polynomial equation that includes the statistical processing of the last Report on Blood Pressure [1]. We also analyzed the effect of height on blood pressure in the 5th to 95th percentile range. Statistical functions generated by computerized program were used.

Results Six monotonic curves of systolic BP for boys representing the 50th, 75th, 90th, 95th, 99th, and 99th +5 percentiles were built (Figure 1). In relation to the table published by the NIH, we confirm use of approximation of the values in the published table by truncation. Considering a tolerance of $1 \mathrm{mmHg}$, the monotonic curve of adjustment for height of the systolic BP for boys does not need any correction in 


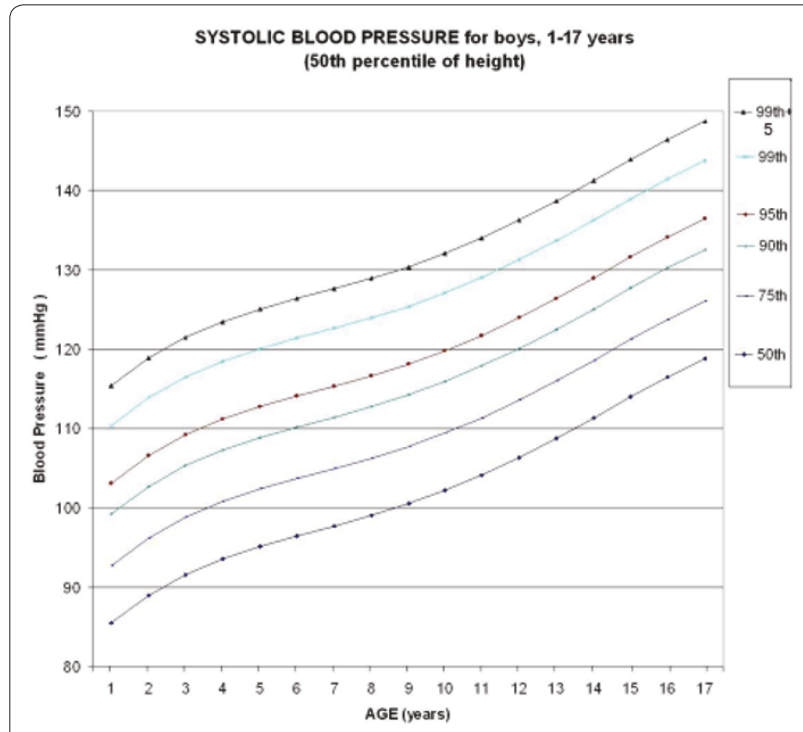

Figure 1 (abstract P18). Update of chart for systolic blood pressure (SBP) based on the last Report on Blood Pressure in 2004 [1], for boys 1 to 17 years old (50th percentile of height). Considering a tolerance of $1 \mathrm{mmHg}$, the curve of adjustment for height of the SBP for boys does not need any correction in the 36.5th to 64.5 th percentile of height.

the 36.5th to 64.5 th percentile of height, but needs maximal correction for the 95th percentile of height $(+3.8 \mathrm{mmHg}$ correction).

Conclusion The adjustment of systolic BP for height is of little significance, and the updated graphic can be used to diagnose high systolic blood pressure for boys. Clinical studies are necessary to determine the systolic BP percentile that better represents clinically significant hypertension.

References

1. National High Blood Pressure Education Program Working Group on High Blood Pressure in Children and Adolescents: the fourth report on the diagnosis, evaluation, and treatment of high blood pressure in children and adolescents. Pediatrics 2004, 114(Suppl 4th Report):555-576.

2. Report of the Second Task Force on Blood Pressure Control in Children - 1987 Task Force on Blood Pressure Control in Children. Pediatrics 1987, 79:1-25.

\section{P19}

Update of the pediatric hypertension graphic adjusted for gender and height percentiles: systolic blood pressure for girls, 1 to 17 years old

HH Shieh, AE Gilio, VHK Koch, DC Raulik, CVranjac, S Fukugava, ER Barreira, EJTroster

University Hospital of Universidade de São Paulo, Vila lara, São Paulo, SP, Brazil Critical Care 2013, 17(Suppl 3):P19 (doi: 10.1186/cc12635)

Introduction Hypertension is the most important preventable risk factor for premature death worldwide. It increases the risk of ischemic heart disease, strokes, peripheral vascular disease, and other cardiovascular diseases, including heart failure, aortic aneurysms, diffuse atherosclerosis, and pulmonary embolism. In childhood, hypertension can be determined according to a table adjusted for height, age and gender [1]. A graphic representation of pediatric hypertension was published in 1987 [2], and no graphic updates have been published since then. The objective of this study was to update the graphic representation of pediatric hypertension.

Methods We used a computerized calculation method to develop high-resolution graphics containing curves with 5,841 points each, to depict the main percentiles associated with high blood pressure for girls from 1 to 17 years old in the 50th percentile of height. Each point represents the calculation of the polynomial equation that includes the statistical processing of the last Report on Blood Pressure [1]. We

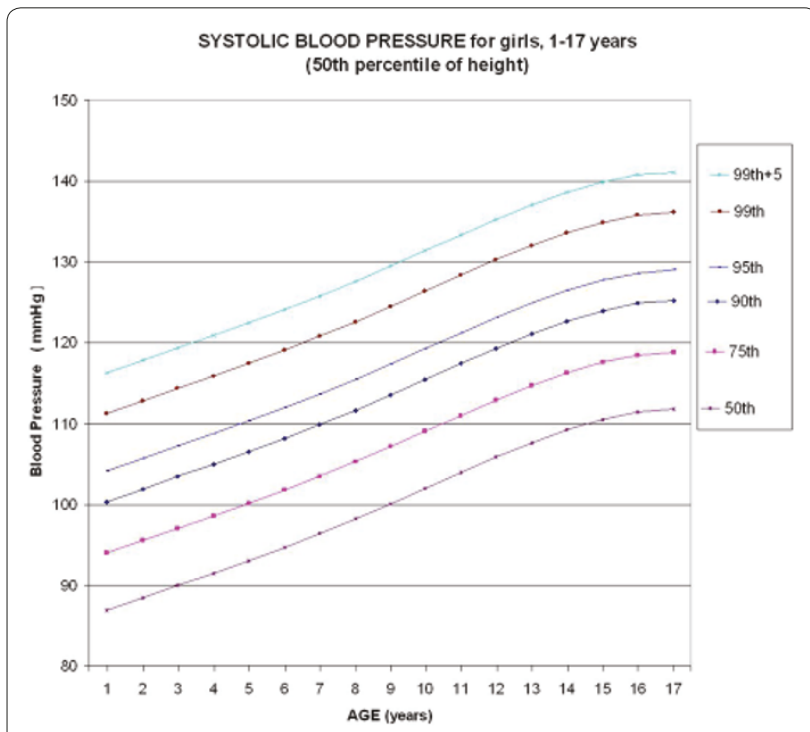

Figure 1 (abstract P19). Update of chart for systolic blood pressure (SBP) based on the last Report on Blood Pressure in 2004 [1], for girls 1 to 17 years old (50th percentile of height). Considering a tolerance of $1 \mathrm{mmHg}$, the curve of adjustment for height of the SBP for girls does not need any correction in the in the 31.5 th to 68.5 th percentile of height.

also analyzed the effect of height on blood pressure in the 5th to 95th percentile range. Statistical functions generated by computerized program were used.

Results Six monotonic curves of systolic BP for girls representing the 50th, 75th, 90th, 95th, 99th, and 99th +5 percentiles were built (Figure 1). In relation to the table published by the $\mathrm{NIH}$, we confirm use of approximation of the values (rounding) in the published table by truncation. Considering a tolerance of $1 \mathrm{mmHg}$, the monotonic curve of adjustment for height of the SBP for girls does not need any correction in the 31.5th to 68.5 th percentile of height, but needs maximal correction for the 5th percentile for height $(-4.7 \mathrm{mmHg}$ correction).

Conclusion The correction of systolic BP for height is of little significance, and this updated graphic can be used to diagnose high systolic blood pressure for girls. Clinical studies are necessary to determine the systolic BP percentile that better represents clinically significant hypertension.

References

1. National High Blood Pressure Education Program Working Group on High Blood Pressure in Children and Adolescents: the fourth report on the diagnosis, evaluation, and treatment of high blood pressure in children and adolescents. Pediatrics 2004, 114(Suppl 4th Report):555-576.

2. Report of the Second Task Force on Blood Pressure Control in Children - 1987 Task Force on Blood Pressure Control in Children. Pediatrics 1987, 79:1-25.

P20

Update of the pediatric hypotension graphic adjusted for gender and height percentiles: diastolic blood pressure for boys, 1 to

17 years old

HH Shieh, ER Barreira, A Bousso, AC Ventura, EJ Troster

University Hospital of Universidade de São Paulo, Vila lara, São Paulo, SP, Brazil

Critical Care 2013, 17(Suppl 3):P20 (doi: 10.1186/cc12636)

Introduction According to the National Heart, Lung, and Blood Institute of the National Institute of Health, hypotension refers to an abnormally low blood pressure (BP). In childhood, hypotension can be determined according to two different definitions: BP below the 5th percentile or below two standard deviations (SDs) of the mean for age and gender [1]. A graphic representation of pediatric hypotension was published in 1977 [2], and no updates have been published since then. 


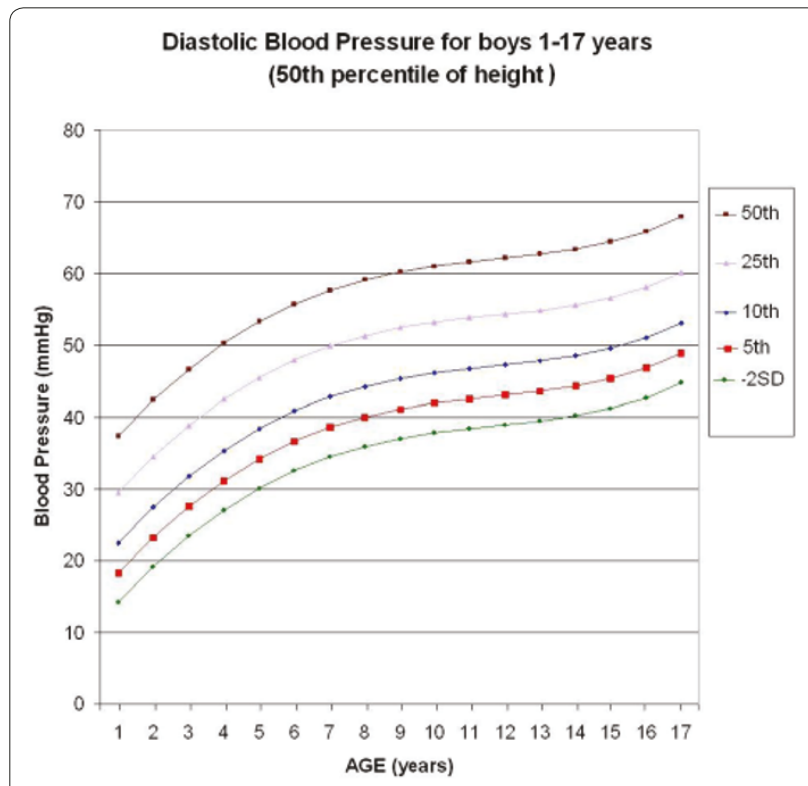

Figure 1 (abstract P20). Graphic update of diastolic blood pressure (DBP) based on the last Report on Blood Pressure in 2004 [3], for boys 1 to 17 years old (50th percentile of height). Considering a tolerance of $1 \mathrm{mmHg}$, the curve of adjustment for height of the DBP for boys does not need any correction in the in the 25.5th to 76 th percentile of height.

The objective of this study was to update the graphic representation of pediatric hypotension.

Methods We used a computerized calculation method to develop high-resolution graphics containing 5,841 points each, to depict the main percentiles associated with low BP for boys from 1 to 17 years old in the 50th percentile of height. Each point represents the calculation of the polynomial equation that includes the statistical processing of the last Report on Blood Pressure [3]. We also analyzed the effect of height on BP in the 5th to 95th percentile range. Statistical functions generated by computerized program were used.

Results Five monotonic curves of diastolic BP for boys representing the 50th, 25th, 10th, 5th, and 2.275th (-2SD) percentiles were built (Figure 1). Considering a tolerance of $1 \mathrm{mmHg}$, the monotonic curve of adjustment for height of the diastolic BP for boys does not need any correction in the 25.5 th to 76 th percentile of height, but needs maximal correction for the 5 th percentile of height $(-2.41 \mathrm{mmHg}$ correction).

Conclusion The correction of diastolic BP for height is of little significance, and this updated graphic can be used to diagnose low diastolic BP for boys. Clinical studies are necessary to determine the SBP percentile that better represents clinically significant hypotension. References

1. Shieh HH, Gilio AE, Barreira ER, Troster EJ, Ventura AMC, Goes PF, Souza DC, Sinimbu Filho JM, Bousso A: Pediatric hypotension: quantification of the differences between the two current definitions. Intensive Care Med 2012, 38(Suppl 1):S0662. doi: 10.1007/s00134-012-2683-0.

2. Blumenthal S, et al: Report of the Task Force on Blood Pressure Control in Children. Pediatrics 1977, 59(Suppl):797.

3. National High Blood Pressure Education Program Working Group on High Blood Pressure in Children and Adolescents: the fourth report on the diagnosis, evaluation, and treatment of high blood pressure in children and adolescents. Pediatrics 2004, 114(Suppl 4th Report):555-576.
P21

Update of the pediatric hypotension graphic adjusted for gender and height percentiles: systolic blood pressure for boys, 1 to 17 years old

HH Shieh, ER Barreira, A Bousso, AC Ventura, EJ Troster

University Hospital of Universidade de São Paulo, Vila lara, São Paulo, SP, Brazil

Critical Care 2013, 17(Suppl 3):P21 (doi: 10.1186/cc12637)

Introduction According to the National Heart, Lung, and Blood Institute of the National Institute of Health, hypotension refers to an abnormally low blood pressure (BP). In childhood, hypotension can be determined according to two different definitions: BP below the 5th percentile or below two standard deviations (SDs) of the mean for age and gender [1]. A graphic representation of pediatric hypotension was published in 1977 [2], and no updates have been published since then. The objective of this study was to update the graphic representation of pediatric hypotension.

Methods We used a computerized calculation method to develop high-resolution graphics containing curves with 5,841 points each, to depict the main percentiles associated with low BP for boys from 1 to 17 years old in the 50th percentile of height. Each point represents the calculation of the polynomial equation that includes the statistical processing of the last Report on Blood Pressure [3]. We also analyzed the effect of height on BP in the 5th to 95th percentile range. Statistical functions generated by computerized program were used.

Results Five monotonic curves of systolic BP for boys representing the 50th, 25th, 10th, 5th, and 2.275th (-2SD) percentiles were built (Figure 1). In relation to the table published by Haque and Zaritzsky [4], the Bland-Altman analysis of the published male systolic BP compared with actual update shows a mean bias of -0.53 , with limits of agreement of -2.01 to $0.96 \mathrm{mmHg}$, which confirms the use of approximation of the values in the published table [4]. Considering a tolerance of $1 \mathrm{mmHg}$, the monotonic curve of adjustment for height of the systolic BP for boys does not need any correction in the 36.5 th to 64.5 th percentile of height, but needs maximal correction for the 95 th percentile of height (+3.8 $\mathrm{mmHg}$ correction).

Conclusion The adjustment of systolic BP for height is of little significance, and the updated graphic can be used to diagnose low systolic BP for boys. Clinical studies are necessary to determine the

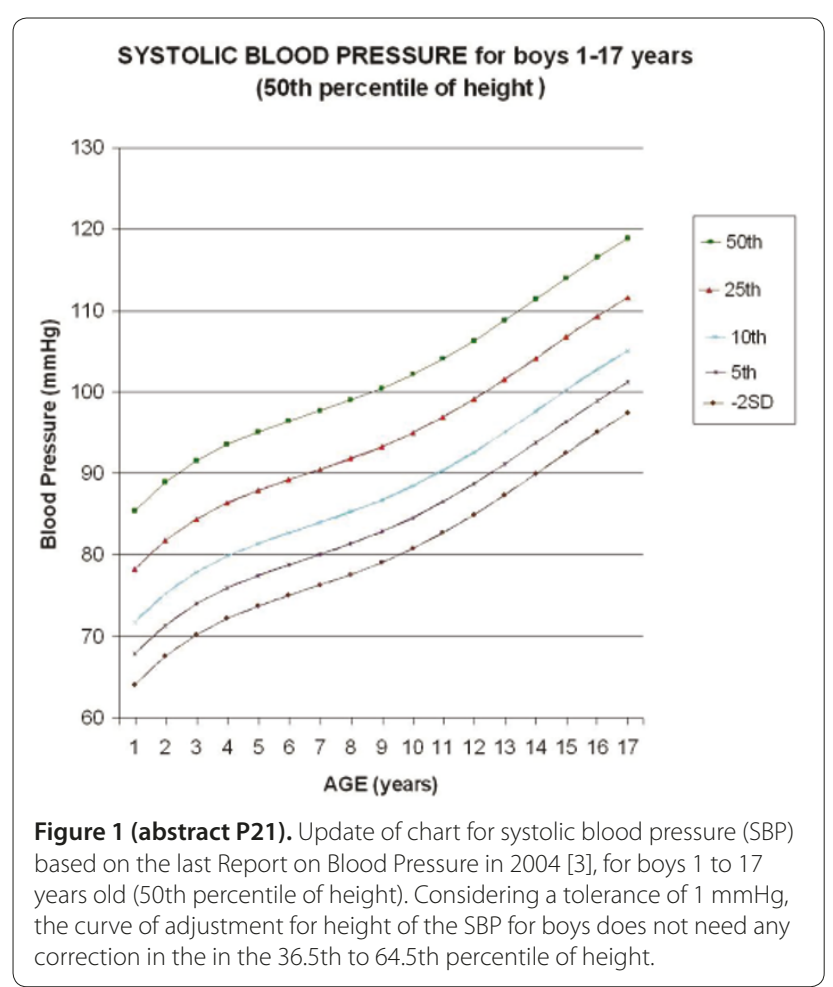


systolic BP percentile that better represents clinically significant hypotension.

\section{References}

1. Shieh HH, Gilio AE, Barreira ER, Troster EJ, Ventura AMC, Goes PF, Souza DC, Sinimbu Filho JM, Bousso A: Pediatric hypotension: quantification of the differences between the two current definitions. Intensive Care Med 2012, 38(Suppl 1):S0662. doi: 10.1007/s00134-012-2683-0

2. Blumenthal S, et al:: Report of the Task Force on Blood Pressure Control in Children. Pediatrics 1977, 59(Suppl):797.

3. National High Blood Pressure Education Program Working Group on High Blood Pressure in Children and Adolescents: the fourth report on the diagnosis, evaluation, and treatment of high blood pressure in children and adolescents. Pediatrics 2004, 114(Suppl 4th Report):555-576.

4. Haque IU, Zaritsky AL: Analysis of the evidence for the lower limit of systolic and mean arterial pressure in children. Pediatr Crit Care Med 2008, 8:138-144.

P22

Update of the pediatric hypotension graphic adjusted for gender and height percentiles: systolic blood pressure for girls, 1 to 17 years old

HH Shieh, ER Barreira, A Bousso, AC Ventura, EJ Troster University Hospital of Universidade de São Paulo, Vila lara, São Paulo, SP, Brazil Critical Care 2013, 17(Suppl 3):P22 (doi: 10.1186/cc12638)

Introduction According to the National Heart, Lung, and Blood Institute of the National Institute of Health, hypotension refers to an abnormally low blood pressure (BP). In childhood, hypotension can be determined according to two different definitions: BP below the 5th percentile or below two standard deviations (SDs) of the mean for age and gender [1]. A graphic representation of pediatric hypotension was published in 1977 [2], and no updates have been published since then. The objective of this study was to update the graphic representation of pediatric hypotension.

Methods We used a computerized calculation method to develop high-resolution graphics containing curves with 5,841 points each, to depict the main percentiles associated with low BP for girls from 1 to 17 years old in the 50th percentile of height. Each point represents

SYSTOLIC BLOOD PRESSURE for girls 1-17 years (50th percentile of height)

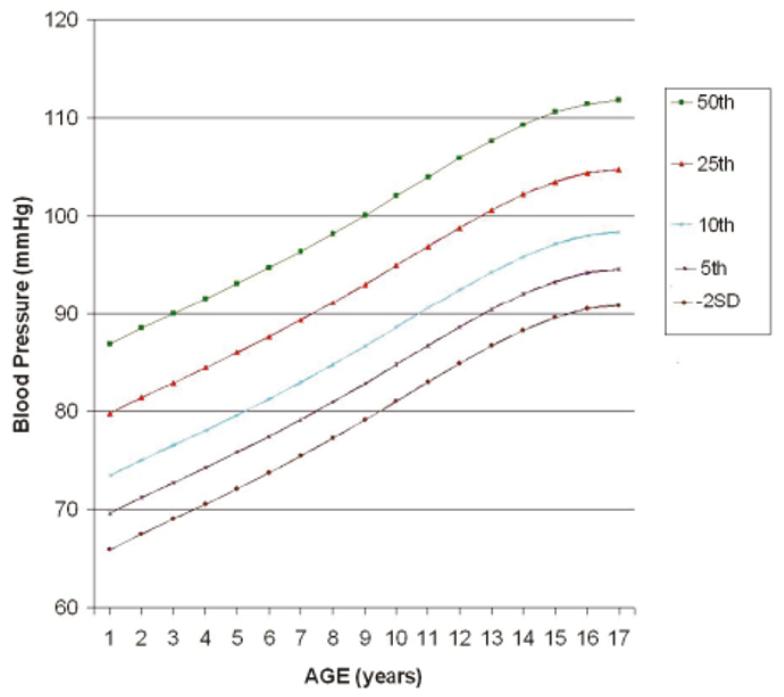

Figure 1 (abstract P22). Update of chart for systolic blood pressure (SBP) based on the last Report on Blood Pressure in 2004 [3], for girls 1 to 17 years old (50th percentile of height). Considering a tolerance of $1 \mathrm{mmHg}$, the curve of adjustment for height of the SBP for girls does not need any correction in the in the 31.5 th to 68.5 th percentile of height. the calculation of the polynomial equation that includes the statistical processing of the last Report on Blood Pressure in 2004 [3]. We also analyzed the effect of height on BP from the 5th to 95th percentile. Statistical functions generated by computerized program were used. Results Five monotonic curves of systolic BP for girls representing the 50th, 25th, 10th, 5th, and 2.275th (-2SD) percentiles were built (Figure 1 ). In relation to the table published by Haque and Zaritzsky [3], the Bland-Altman analysis of the published female systolic BP compared with actual update shows a mean bias of -0.61 , with limits of agreement from -2.12 to $0.9 \mathrm{mmHg}$, which confirms the use of approximation of the values in the published table [3]. Considering a tolerance of $1 \mathrm{mmHg}$, the monotonic curve of adjustment for height of the SBP for girls does not need any correction in the 31.5th to 68.5th percentile of height, but needs maximal correction for the 5th percentile for height (-4.7 $\mathrm{mmHg}$ correction).

Conclusion The correction of systolic BP for height is of little significance, and this updated graphic can be used to diagnose low systolic BP for girls. Clinical studies are necessary to determine the systolic BP percentile that better represents clinically significant hypotension.

References

1. Shieh HH, Gilio AE, Barreira ER, Troster EJ, Ventura AMC, Goes PF, Souza DC, Sinimbu Filho JM, Bousso A: Pediatric hypotension: quantification of the differences between the two current definitions Intensive Care Med 2012, 38(Suppl 1):S0662. doi: 10.1007/s00134-012-2683-0

2. Blumenthal S, et al:: Report of the Task Force on Blood Pressure Control in Children. Pediatrics 1977, 59(Suppl):797.

3. National High Blood Pressure Education Program Working Group on High Blood Pressure in Children and Adolescents: the fourth report on the diagnosis, evaluation, and treatment of high blood pressure in children and adolescents. Pediatrics 2004, 114(Suppl 4th Report):555-576.

4. Haque IU, Zaritsky ALL Analysis of the evidence for the lower limit of systolic and mean arterial pressure in children. Pediatr Crit Care Med 2008, 8:138-144

P23

Update of the pediatric hypotension graphic adjusted for gender and height percentiles: diastolic blood pressure for girls, 1 to 17 years old

HH Shieh, ER Barreira, A Bousso, AC Ventura, EJ Troster

University Hospital of Universidade de São Paulo, Vila lara, São Paulo, SP, Brazil Critical Care 2013, 17(Suppl 3):P23 (doi: 10.1186/cc12639)

Introduction According to the National Heart, Lung, and Blood Institute of the National Institute of Health, hypotension refers to an abnormally low blood pressure (BP). In childhood, hypotension can be determined according to two different definitions: BP below the 5th percentile or below two standard deviations (SDs) of the mean for age and gender [1]. A graphic representation of pediatric hypotension was published in 1977 [2], and no updates have been published since then. The objective of this study was to update the graphic representation of pediatric hypotension.

Methods We used a computerized calculation method to develop high-resolution graphics containing curves with 5,841 points each, to depict the main percentiles associated with low BP for girls from 1 to 17 years old in the 50th percentile of height. Each point represents the calculation of the polynomial equation that includes the statistical processing of the last Report on Blood Pressure in 2004 [3]. We also analyzed the effect of height on BP from the 5th to 95th percentile. Statistical functions generated by computerized program were used. Results Five monotonic curves of diastolic BP for girls representing the 50th, 25th, 10th, 5th, and 2.275th (-2SD) percentiles were built (Figure 1). Considering a tolerance of $1 \mathrm{mmHg}$, the monotonic curve of adjustment for height of the DBP for girls does not need any correction in the 16th to 78.5th percentile of height, but needs maximal correction for the 95th percentile of height $(+2.08 \mathrm{mmHg}$ correction).

Conclusion The correction of female diastolic BP for height is of minimal significance, and this updated graphic can be used to diagnose low diastolic BP for girls. Clinical studies are necessary to determine the diastolic BP percentile that better represents clinically significant hypotension. 


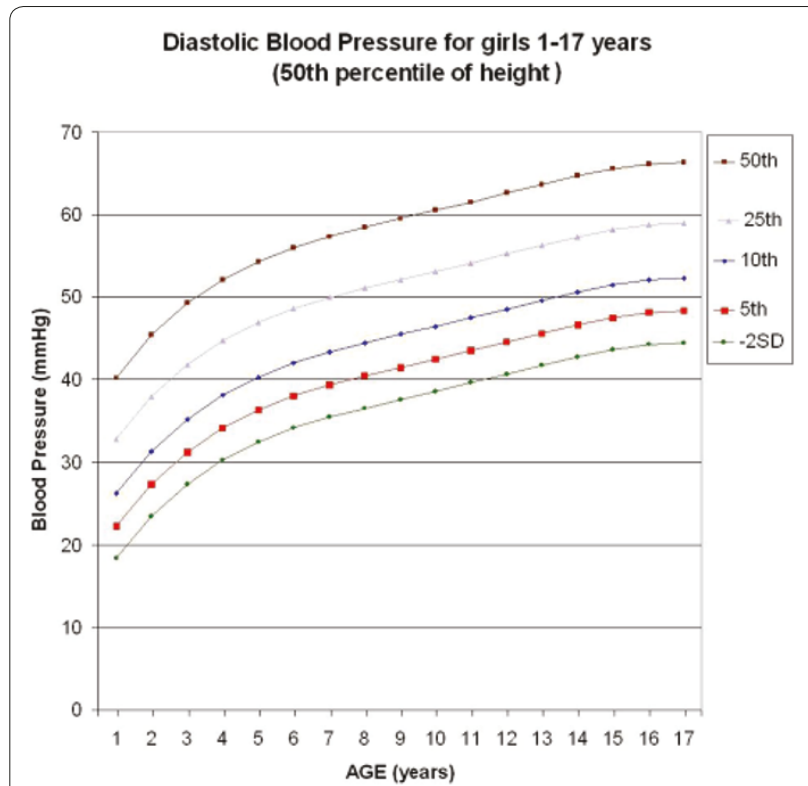

Figure 1 (abstract P23). Graphic update of diastolic blood pressure (DBP) based on the last Report on Blood Pressure in 2004 [3], for girls 1 to 17 years old (50th percentile of height). Considering a tolerance of $1 \mathrm{mmHg}$ the curve of adjustment for height of the DBP for girls does not need any correction in the 16th to 78.5th percentile of height.

\section{References}

1. Shieh HH, Gilio AE, Barreira ER, Troster EJ, Ventura AMC, Goes PF, Souza DC, Sinimbu Filho JM, Bousso A: Pediatric hypotension: quantification of the differences between the two current definitions Intensive Care Medicine 2012, 38(Suppl 1):S0662. doi: 10.1007/s00134-012-2683-0.

2. Blumenthal S, et al:: Report of the Task Force on Blood Pressure Control in Children. Pediatrics 1977, 59(Suppl):797.

3. National High Blood Pressure Education Program Working Group on High Blood Pressure in Children and Adolescents: the fourth report on the diagnosis, evaluation, and treatment of high blood pressure in children and adolescents. Pediatrics 2004, 114(Suppl 4th Report):555-576.

\section{P24}

Vasopressin and terlipressin in adult vasodilatory shock: a systematic review and meta-analysis of nine randomized controlled trials

VGM Pereira, A Serpa Neto, SO Cardoso, JA Manetta, DC Espósito, $M$ de Oliveira Prado Pasqualucci

ABC Medical School (FMABC), Príncipe De Gales, Santo André, SP, Brazil

Critical Care 2013, 17(Suppl 3):P24 (doi: 10.1186/cc12640)

Introduction Catecholamines are the most used vasopressors in vasodilatory shock. However, the development of adrenergic hyposensitivity and the subsequent loss of catecholamine pressor activity necessitate the search for other options. Our aim was to evaluate the effects of vasopressin and its analogue terlipressin compared with catecholamine infusion alone in vasodilatory shock.

Methods Systematic review and meta-analysis of publications between 1966 and 2011. The Medline and CENTRAL databases were searched for studies on vasopressin and terlipressin in critically ill patients. The metaanalysis was limited randomized controlled trials evaluating the use of vasopressin and/or terlipressin compared with catecholamine in adult patients with vasodilatory shock. The assessed outcomes were: overall survival, changes in the hemodynamic and biochemical variables, a decrease of catecholamine requirements, and adverse events.

Results Nine trials covering 998 participants were included. A metaanalysis using a fixed-effect model showed a reduction in norepinephrine requirement among patients receiving terlipressin or vasopressin infusion compared with control (standardized mean difference, -1.58 (95\% Cl, -1.73 to -1.44$) ; P<0.0001)$. Overall, vasopressin and terlipressin, as compared with norepinephrine, reduced mortality (relative risk (RR): 0.87 ( 0.77 to 0.99$) ; P=0.04)$. Vasopressin compared with norepinephrine decreased mortality in adult patients (RR: $0.87(0.76$ to 1.00$) ; P=0.05)$ and in patients with septic shock (42.5\% vs. $49.2 \%$, respectively; RR, 0.87 ( $95 \%$ $\mathrm{Cl}, 0.75$ to 1.00 ); $P=0.05$; number needed to treat, 1 to 15 ). There was no difference in adverse events between the vasopressin and control groups (RR: 0.98 (0.65 to 1.47 ); $P=0.92$ ).

Conclusion Vasopressin use in vasodilatory shock is safe, associated with reduced mortality, and facilitates weaning of catecholamines. In patients with septic shock, vasopressin compared with norepinephrine may also decrease mortality.

\section{Infection}

P25

A phase 1 study of intravenously administered TR-701 FA in subjects with advanced renal impairment

S Flanagan', D Morris², T Boyea', H Dreskin', SL Minassian', H Alcorn³. T Marbury ${ }^{4}$, M Abdelhameed ${ }^{2}$, E Fang ${ }^{1}$, P Prokocimer ${ }^{1}$

${ }^{1}$ Trius Therapeutics, San Diego, CA, USA: ${ }^{2}$ Covance, Madison, WI, USA: ${ }^{3}$ DaVita, Minneapolis, MN, USA; ${ }^{4}$ Orlando Clinical Research Center, Orlando, FL, USA Critical Care 2013, 17(Suppl 3):P25 (doi: 10.1186/cc12641)

Introduction The objective of the study was to characterize the safety of tedizolid phosphate (TR-701 FA) and the pharmacokinetics of tedizolid (TR-700), its microbiologically active moiety, in subjects with advanced renal impairment (eGFR $<30 \mathrm{ml} /$ minute $/ 1.73 \mathrm{~m}^{2}$, not on dialysis) compared with matched subjects with normal renal function (eGFR $\geq 80.0 \mathrm{ml} / \mathrm{minute} / 1.73 \mathrm{~m}^{2}$ ).

Methods Eight subjects with advanced renal impairment and eight matched controls (by age, gender, and body mass index) received a single intravenous infusion of $200 \mathrm{mg}$ TR-701 FA. Serial plasma PK samples were collected from pre-dose through 72 hours postdose. Plasma samples were analyzed for TR-700 and the following pharmacokinetic parameters were calculated: $C_{\max ^{\prime}} \mathrm{t}_{\text {max }}, \mathrm{AUC}_{0-\infty^{\prime}}, \mathrm{AUC}_{0-\mathrm{t}^{\prime}}$ $\lambda_{z^{\prime}} \mathrm{CL}_{\mathrm{sys}}$ and plasma $\mathrm{t}_{1}$

Results Baseline eGFR ranged from 7 to $28 \mathrm{ml} /$ minute $/ 1.73 \mathrm{~m}^{2}$ (including three subjects with eGFR $<15 \mathrm{ml} /$ minute $/ 1.73 \mathrm{~m}^{2}$ ). The pharmacokinetics of TR-700 was essentially unchanged in subjects with advanced renal impairment relative to a matched control group. Approximately $8 \%$ lower AUC and nearly identical $C_{\max }$ values were observed in renal impaired subjects relative to matched controls, and other pharmacokinetic parameters were also similar between groups. See Table 1

Table 1 (abstract P25). Pharmacokinetic parameters of tedizolid in control and renal impaired subjects

\begin{tabular}{|c|c|c|c|c|c|}
\hline \multirow[b]{2}{*}{ TR-700 } & \multicolumn{3}{|c|}{ Geometric mean (GM) } & \multicolumn{2}{|c|}{$90 \% \mathrm{Cl}$} \\
\hline & $\begin{array}{l}\text { Advanced } \\
\text { renal } \\
\text { impairment }\end{array}$ & Control & GM ratio & Lower & Upper \\
\hline $\mathrm{AUC}_{0-\mathrm{t}}(\mu \mathrm{g} \cdot \mathrm{hour} / \mathrm{ml})$ & 28.4 & 30.7 & 0.93 & 0.70 & 1.23 \\
\hline $\mathrm{AUC}_{0-\infty}(\mu \mathrm{g} \cdot$ hour$/ \mathrm{ml})$ & 28.7 & 31.0 & 0.92 & 0.70 & 1.23 \\
\hline$C_{\max }(\mu \mathrm{g} / \mathrm{ml})$ & 3.01 & 3.02 & 0.99 & 0.78 & 1.27 \\
\hline
\end{tabular}

Conclusion The TR-700 plasma pharmacokinetic results from this study provide support that no dose adjustment is needed in subjects with advanced renal impairment.

Acknowledgement Trial registration: NCT01452828.

P26

Abstract withdrawn 
P27

Parenteral colistin for the treatment of severe infections by multidrug-resistant Gram-negative bacteria

C Grion, MT Tanita, CM Dantas de Maio Carrilho, JP Garcia, J Festti, LTQ Cardoso, F Chiquetti, MM Kanehissa, CC Branco Lopes, D Blum, $\checkmark$ Anami, AR Ruiz, PA Rossatto

Hospital Universitário - Universidade Estadual de Londrina, Vila Operária, Londrina, PR, Brazil

Critical Care 2013, 17(Suppl 3):P27 (doi: 10.1186/cc12643)

Introduction This study aimed to describe the use of colistin to treat nosocomial infections caused by multidrug-resistant Gram-negative bacteria and to identify risk factors associated with acute kidney injury and mortality in a single center.

Methods This prospective longitudinal study evaluates critically ill patients with nosocomial infections caused by multidrug-resistant Gram-negative bacteria. All adult patients requiring treatment with intravenous colistin (colistimethate sodium) from January to December 2008 were considered eligible for the study. Data include demographics, diagnosis, duration of treatment with colistin and 30-day mortality. Data were analyzed using MedCalc 12 (MedCalc Software, Belgium).

Results Colistin was used to treat an infection in 109 (13.81\%) of the 789 patients admitted to the ICU. Colistin treatment started an average of 10 (6 to 14.25) days after admission to the ICU. The 30-day mortality observed in these patients was $71.56 \%$. Twenty-four (22.02\%) patients developed acute kidney injury, and 11 of these patients required dialysis. The variables independently associated with the presence of acute kidney injury were concomitant use of vancomycin (OR 3.10; $95 \% \mathrm{Cl} 1.10$ to $8.69 ; P=0.032$ ) and vasopressors (OR 4.22; $95 \% \mathrm{Cl}$ 1.23 to $14.43 ; P=0.022$ ). Age (OR $1.03 ; 95 \% \mathrm{Cl} 1.00$ to $1.05 ; P=0.027$ ) and vasopressor use (OR 12.48; $95 \% 4.49$ to $34.70 ; P<0.001)$ were independent risk factors for death in the logistic-regression model. Conclusion The concomitant use of vancomycin was associated with increased risk of acute kidney injury. Shock and vasopressors were associated with increased risk for acute kidney injury and death.

P28

Abstract withdrawn

P29

Vancomycin dose adjustment in severe burn patients based on trough level for drug effectiveness against pathogens at $1 \mathrm{mg} / \mathrm{l}$ minimum inhibitory concentration

JM Silva Jr', AM Oliveira', EV Campos', DS Gomez', MC Ferreira', CS Giraud $^{2}$, CV Silva Jr2, SRCJ Santos ${ }^{2}$

'Burn Unit/Plastic Surgery Division of Clinics Hospital, Medical School, University of Sao Paulo, Butantã, São Paulo, SP, Brazil; ${ }^{2}$ School of

Pharmaceutical Sciences, University of Sao Paulo, Butantã, São Paulo, SP, Brazil

Critical Care 2013, 17(Suppl 3):P29 (doi: 10.1186/cc12645)

Introduction Vancomycin is usually prescribed to severe burn patients with sepsis from the intensive care burn unit (ICBU) for control of hospital infection. The objective of this study was to evaluate the contribution of dose regimen adjusted in burn patients with renal function preserved based on drug plasma concentration at the trough and pharmacokinetic-pharmacodynamic (PK/PD) correlation.

Methods Sixty severe burn patients with documented Gram-positive nosocomial infection from the ICBU were enrolled in a prospective cohort study, and the period of inclusion was 2 years; the protocol was approved by the hospital's ethical committee. Patients of both genders (43 male/17 female) with preserved renal function were investigated (176 sets). The vancomycin dose regimen was initially $2 \mathrm{~g}$ daily for the control of infection in burn patients with sepsis. Pharmacotherapeutic follow-up was performed by a serial blood sample collection ( $2 \mathrm{ml}$ each) for drug plasma measurements [1]. Drug effectiveness was based on the parameter $\mathrm{AUC}_{\mathrm{sS0}-24} / \mathrm{MIC}>400$ [2]; $\mathrm{AUC}_{\mathrm{ss} 0-24}$ was the area under the curve (plasma concentration vs. time) integrated up to 24 hours, and the minimum inhibitory concentration (MIC) from in vitro antimicrobial susceptibility testing performed in the hospital [2]. Dose adjustment was required and the daily dose was increased to reach trough levels $>10 \mu \mathrm{g} / \mathrm{ml}$ and AUC/MIC $>400$.

Results Characteristics of patients investigated were (mean $\pm \mathrm{SD}$ ): $38.9 \pm 14.1$ years; $70.0 \pm 10.6 \mathrm{~kg}, 28.0 \pm 19.0 \%$ total burn surface area. Thermal injury occurred in $51 / 60$ patients, and inhalation injury occurred in $54.9 \%$ of them, versus electrical injury reported in $9 / 60$ patients; renal function was monitored by serum creatinine $(0.72 \pm 0.29 \mathrm{mg} / \mathrm{dl})$ and creatinine clearance (153.7 $\pm 70.4 \mathrm{ml} /$ minute). Significant increase was chosen by comparison of the initial dose against adjusted dose according to trough levels $>10 \mathrm{~g} / \mathrm{ml}$ and also AUC/MIC $>400$ (Table 1).

Table 1 (abstract P29). Daily dose medians, trough and PK/PD data

\begin{tabular}{lccc}
\hline Parameter & Initial dose & Adjusted dose & $P$ value \\
\hline Daily dose $(\mathrm{mg} / \mathrm{kg} /$ day $)$ & 28.6 & 42.9 & 0.01 \\
Trough levels $(\mu \mathrm{g} / \mathrm{ml})$ & $7.1(3.6$ to 13.3$)$ & $16.0(12.0$ to 23.3$)$ & $<0.001$ \\
AUC/MIC (MIC: $1 \mathrm{mg} / \mathrm{l})$ & $436(248$ to 659$)$ & $648(467$ to 942$)$ & 0.02 \\
\hline
\end{tabular}

Data presented as median ( 25 to $75 \%$ percentile).

Conclusion The initial dose recommended for vancomycin must be increased according to trough levels and also AUC/MIC to achieve the PK/PD target in burn patients with preserved renal function.

Acknowledgement Foundation for Research State of Sao Paulo/SP, Brazil (FAPESP).

References

1. Lopéz KJV, Bertoluci DF, Vicente KM, Dell'Aquilla AM, Santos SRCJ: Simultaneous determination of cefepime, vancomycin and imipenem in human plasma of burn patients by high performance liquid chromatography. J Chromatogr B 2007, 860:241-245.

2. Revilla N, Marín-Suárez A, Pérez MP, González FM, Gatta MMF: Vancomycin dosing assessment on intensive care unit patients based on a population pharmacokinetic/pharmacodynamic simulation. Br J Clin Pharmacol 2010, 70:201-212.

\section{Nephrology}

\section{P30}

Acute kidney injury according to RIFLE criteria in an ICU: incidence and mortality impact

AR Santana, FF Amorim, FB Soares, LG de Souza Godoy,

L de Jesus Almeida, TA Rodrigues, G Menezes de Andrade Filho, TA Silva,

JL de Souza, KCM Ogliari, PN Ferreira Jr, APP Amorim, EB de Moura,

JA de Araújo Neto, M de Oliveira Maia

Unidade de Terapia Intensiva Adulto do Hospital Santa Luzia, Asa Sul, Brasília, DF, Brazil

Critical Care 2013, 17(Suppl 3):P30 (doi: 10.1186/cc12646)

Introduction Acute kidney injury (AKI) is a very common condition in hospitalized patients, especially in ICUs. It is also closely related to adverse patient outcomes, mortality rates as high as three-quarters and as many as $13 \%$ need of renal support after hospital discharge. A systematic review demonstrated a close correlation between AKI according to the RIFLE criteria and mortality. The objective of this study was to evaluate the incidence of AKI according to the RIFLE criteria and the impact of each category on mortality in an ICU.

Methods A retrospective cohort study was conducted with patients admitted to the adult ICU of Hospital Santa Luzia, Brasilia, DF, Brazil, in the period of 6 months. Patients were categorized as Risk (R), Injury (I), Failure (F), or without AKI according to RIFLE criteria. Patients with a previous diagnosis of chronic kidney disease were excluded.

Results A total of 626 patients were included. Average age was $60 \pm 20$ years, 326 were male $(50.8 \%)$, APACHE II was $9 \pm 6,67.1 \%$ $(n=326)$ were nonsurgical, and the mortality rate was $12.3 \%(n=77)$. According to RIFLE criteria, 148 had AKI. Eighty-three patients were classified as $\mathrm{R}(13.3 \%$, mortality rate of $21.7 \%), 43$ as I (6.9\%, mortality rate of $53.5 \%)$, and 22 patients as $\mathrm{F}(3.5 \%$, mortality rate of $54.5 \%)$. The relative risk (RR) of death in patients classified as $\mathrm{R}$ was $2.72(95 \% \mathrm{Cl}$ : 1.26 to 4.09 ), I was 11.27 (95\% Cl: 5.81 to 21.83), and F was 9.91 (95\% 


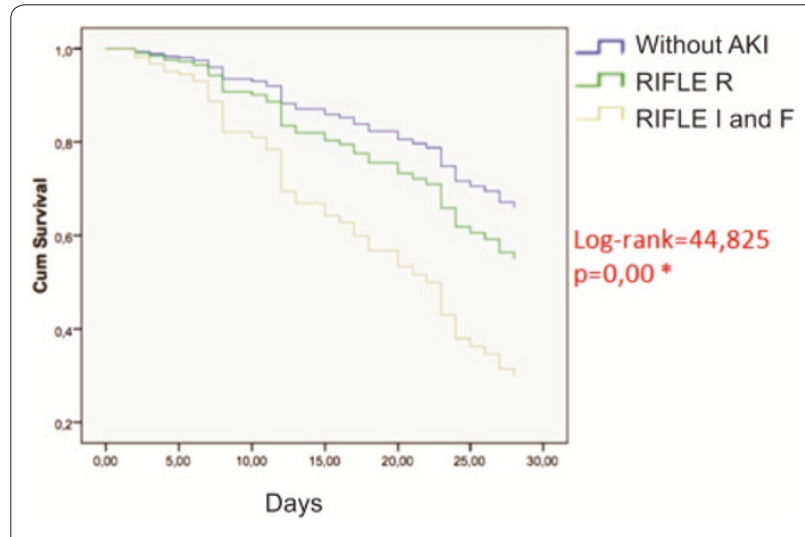

Figure 1 (abstract P30).

$\mathrm{Cl}: 4.14$ to 23.94$)$. Analyzing all patients with AKI, the RR of death was 11.22 (95\% Cl: 6.57 to 19.17). Eight (1.3\%) patients underwent renal replacement therapy during ICU hospitalization, and mortality in these patients was $75 \%$ (RR: $23.11,95 \% \mathrm{Cl} 4.58$ to 116.71 ). Significant difference was observed in the Kaplan-Meier survival curves of the patients with or without AKI at 28 days $(P=0.00)$. See Figure 1.

Conclusion AKI according to RIFLE criteria was associated with an increased mortality for all categories, mainly in patients with criteria to injury and acute kidney failure, and notably those who needed renal replacement therapy.

References

1. Vincent JL, et al:: Crit Care Med 2006, 34:344-353.

2. Uchino et al: JAMA 2005, 294:831-838.

3. Ricci Z, et al:: Kidney Int 2008, 73:538-546.

P31

Analysis of cytokine profile and heme metabolism byproducts after hemorrhagic stroke

CR Shinotsuka, FA Bozza

Hospital Copa Dor, Copacabana, Rio de Janeiro, RJ, Brazil

Critical Care 2013, 17(Suppl 3):P31 (doi: 10.1186/cc12647)

Introduction Intracerebral hemorrhage is a fatal disease, accounting for about $15 \%$ of deaths from stroke. Local and systemic inflammatory response associated with hemoglobin byproducts seems to have a causal role in neuronal death and ICH prognosis. The objective of this study was to investigate the relationship of cytokine profile and hemoglobin degradation products with brain injury severity and prognosis.

Methods We developed a prospective cohort study conducted in three tertiary hospitals. All ICH patients with hemoventricle and external ventricular device (EVD) inserted who were admitted to the neurocritical care unit between 2008 and 2011 were included. We collected blood and cerebrospinal fluid (CSF) from the EVD on days 1 , 2, 3, 5, and 7 after ICH for measurement of C-reactive protein, cytokines, heme, hemoglobin, cytometry, hemopexine, haptoglobin, enolase, and $\mathrm{s}-100 \mathrm{~B}$ concentration. A multiplex analysis was performed to evaluate levels of 17 cytokines. CT scans were evaluated for hematoma and hemoventricle volume. Primary outcome was death in 7 days. This study was approved by the ethics committee of all participating institutions.

Results Fifteen patients were included. Median age was 59 years (IQR: 55 to 65), six patients (40\%) were male. Median Glasgow Coma Scale was 7 (6 to 9), APACHE II score was 22 (IQR: 15 to 25) and SAPS III was 43 (IQR: 32 to 53). Five patients had hemorrhagic stroke and 10 subarachnoid hemorrhage. Overall mortality in 7 days was $40 \%$ (six patients) and in 28 days was $66.6 \%$ (10 patients). Median hematoma volume was $10.53 \mathrm{ml}$ (IQR: 5.42 to 31.75 ) and hemoventricle volume was 8.86 (IQR: 0 to 27.08). Plasmatic iron concentration was higher in nonsurvivors than in survivors $(496.04 \times 58.5 \mathrm{mg} / \mathrm{dl}, P=0.05) 24$ hours after the event. CSF cytometry and lymphocyte count was more increased in nonsurvivors than in survivors (WBC count: $247.5 \times 3$ cells/ $\mathrm{ml}, P=0.01$ and lymphocyte count: $179 \times 5$ cells $/ \mathrm{ml}, P=0.01) 24$ hours after the event. Plasmatic IL-2, IL-6, IL-8, and IL-12 levels are higher in nonsurvivors than in survivors. CSF IL-4 levels were higher in survivors and CSF IL-17 levels were higher in nonsurvivors than in survivors. There was no correlation between plasmatic or CSF levels of enolase and S100-B with mortality. Plasmatic hemopexine and haptoglobin levels also do not seem to be associated with survival.

Conclusion A systemic and local proinflammatory response is associated with higher mortality in patients with hemorrhagic stroke. CSF IL-4 higher concentrations are associated with better prognosis.

P32

Characterization of potential donors of multiple organs and tissues reported in Maranhão from 2009 to 2012

TM Correa, G Castro, JS da Silva, JN Bacelar

Hospital Centro Médico, Monte Castelo, São Luís, MA, Brazil

Critical Care 2013, 17(Suppl 3):P32 (doi: 10.1186/cc12648)

Introduction Thousands of lives worldwide are improved annually with transplant and donation of organs and tissues. Human organ transplant was one of the greatest medical advances in the 20th century, with a success rate between 80 and $90 \%$. The Brazilian National Transplantation System coordinates and regulates perhaps the largest public transplantation program worldwide. However, family refusal represents the biggest obstacle to the donation process. It is one of the main factors responsible for the shortage of organs and tissues for transplantation. The objectives of this study were to characterize potential donors reported to the Central of Notification, Captation and Distribution of Organs and Tissues in Maranhão (CNCDO-MA) between January 2009 and September 2012, to evaluate the frequency of potential donors converted into effective donors, and to identify the captured organs in effective donors.

Methods A descriptive, retrospective study and quantitative analysis from CNCDO-MA reports, using a standardized data collection form. The inclusion criteria was suspected brain death (BD), with at least the first clinical examination for BD protocol performed - potential donor, notified to CNCDO-MA between January 2009 and September 2012. The exclusion criteria were the notifications in which the cause of death was unknown or supplementary test results were inconsistent with BD. Results A total of 345 notifications were analyzed. The majority were male $(63.2 \%)$, with an average age of $37.85( \pm 18.47)$ years. The most incident causes of BD were traumatic brain injury (39.7\%) and hemorrhagic stroke $(36.2 \%)$. Arteriography was the predominant supplementary examination (14.5\%) to complete the BD protocol. The potential donors converted into effective donors were $14.8 \%$. The amount of collected organs in effective donors was 50 corneas, 29 kidneys, six hearts and one liver.

Conclusion The notification and donation of organs and tissues rates are lower in Maranhão than in Brazil. The integration of multidisciplinary teams is necessary for the responsibility of maintaining potential donors and mandatory BD notification, regardless of the chance of organ donations, which can improve the rates in our state.

P33

Comparison between APACHE II and POSSUM 2 scores in neurosurgical patients admitted to an ICU in Fortaleza, Brazil

AA Peixoto Jr', , MNR Teixeira Henderson ${ }^{3}$, CAR Feijón ${ }^{3}$, VN Araújo3,

ZC Teixeira ${ }^{3}$, RE Magalhães ${ }^{1,3}$, FA de Meneses , $^{1,3}$

'Centro Universitário Christus - Unichristus, Papicu, Fortaleza, CE, Brazil;

${ }^{2}$ Hospital Universitário Walter Cantídio - UFC, Papicu, Fortaleza, CE, Brazil;

${ }^{3}$ Hospital Geral De Fortaleza - SESA, Papicu, Fortaleza, CE, Brazil

Critical Care 2013, 17(Suppl 3):P33 (doi: 10.1186/cc12649)

Introduction The need to stratify surgical risk is essential to assess the quality of care of patients undergoing intervention. The objective of this study was to compare APACHE II and POSSUM 2 severity scores in patients undergoing neurosurgery, in the immediate postoperative period. 
Methods An observational prospective study with 155 patients admitted consecutively to the ICU of a tertiary hospital in Fortaleza, Brazil, from December 2011 to June 2012, after neurosurgical intervention.

Results The population analyzed showed an average age of $48.0 \pm 15.8$ years, predominantly female $(55.3 \%)$. Elective surgeries were more prevalent $(92.1 \%)$, especially aneurysm clipping $(14.6 \%)$ and resection of neoplasm (64.2\%). At admission, 45 patients (27.6\%) had at least one organ dysfunction. The APACHE II score mean was $9.7 \pm 5.1$, corresponding to the mean of predicted mortality of $14.0 \pm 10.7 \%$. The POSSUM 2 score showed a trend to be higher in patients that died, the mean of the physiological score being 17.7 (20.0 IQ: 13.8 to 24.3 vs. 16.5 IQ: 14 to $20, P=0.416$ ) and the mean of surgical score being 11.2 (13.0 IQ: 10.5 to 14.3 vs. 10.0 IQ: 9 to $12, P=0.06$ ), corresponding to a mean of predicted mortality of $7.3 \%$ (6.9 IQ: 3.9 to 14.6 vs. 4.0 IQ: 2.6 to $6.4, P=0.08$ ) and average morbidity of $29.6 \%$ (37.0\% IQ: 21.9 to 61.8 vs. $21.1 \%$ IQ: 14.6 to $34.3, P=0.06$ ). The mortality rate was $6.57 \%$, generating a standardized mortality rate of 0.47 for the APACHE II score and 0.90 for the POSSUM 2 score.

Conclusion Our study suggests that the POSSUM 2 score could be a useful tool in predicting mortality in neurosurgical patients admitted to an ICU. It was more accurate to identify the real mortality than the APACHE II score.

\section{P34}

\section{Electroncephalography in an ICU: indications and results}

SP Cavalcanti, G Castro, L Pineiro e Pinho, RCC Lobo Ferreira,

$\checkmark$ de Carvalho Bacelar, R Silva de Carvalho, SM Pereira de Castro,

P da Silva Sousa Carvalho

Ceuma University, Renascença II, São Luís, MA, Brazil

Critical Care 2013, 17(Suppl 3):P34 (doi: 10.1186/cc12650)

Introduction Evaluation electroencephalography (EEG) in the ICU provides a noninvasive measure at the bedside of brain function in critically ill patients. The objectives of this study were to determine the main indications for electroencephalographic monitoring in ICUs, the possible findings, and clinical outcome of patients.

Methods This was a retrospective study analyzing the records of patients who underwent EEG study from October 2011 to October 2012. Data collection was performed with the aid of a standardized form, prepared by the authors, which consisted of the following variables: age, sex, indications for performing EEG, ICU admission diagnosis, electroencephalographic findings, and clinical outcome of the patient. After collection, the data were tabulated using Excel 2007. Later they were exposed in descriptive tables and the statistical analysis was performed using the Statistical Program 7.0, considering a significance level of 0.05 .

Results We analyzed 35 EEGs in 29 patients; five underwent more than one examination. Of these patients, 17 (58.6\%) were male and $12(41.4 \%)$ were female. The mean age was $64.59 \pm 17.91$. With regard to clinical outcome, we observed that $79.3 \%$ of those assessed were epileptiform discharges and only $20.7 \%$ died. We found that acute neuronal injury was the most observed clinical diagnosis, accounting for $28.5 \%$ (AVEH, $17.1 \%$ and AVEI, 11.4\%), followed by TBI (17.1\%), severe sepsis $(17.1 \%)$, toxic-metabolic encephalopathy $(11.4 \%)$, status epilepticus (11.4\%) and others (14.3\%). No statistical significance occurred when we correlated diagnosis and mortality. The EEG was performed if the patient had an altered level of consciousness (62.7\%), seizures $(25.7 \%)$ or a combination of altered consciousness and convulsion (8.6\%). Coma was present in $11.4 \%$ and only $2.9 \%$ had EEG evaluation for post PCR. The presence of epileptiform discharges was identified in $11(31.4 \%)$, although most did not show this pattern (24, $68.6 \%)$. Of the 35 reports, seven (20\%) had electrographic status and among these four (11.4\%) had an epileptiform pattern while three (8.57\%) met periodic pattern criteria. All patients had electrographic status resolution of seizures and were discharged.

Conclusion EEG shows great applicability in ICUs, guiding sound decisions in patients with impaired consciousness.
P35

Evaluation of new variables to predict delirium outcome

JPLM de Carvalho', R Alvim', JCS Martins', C Bouza', P Zenaide²,

R Zantieff', B Pondé', D Amorim', LC Quarantini' ${ }^{3}$, D Gusmao-Flores ${ }^{3}$

'Faculdade de Medicina da Bahia, Universidade Federal da Bahia, Salvador, BA, Brazil; '2Escola Bahiana de Medicina e Saúde Pública, Salvador, BA, Brazil;

${ }^{3}$ University Hospital Prof. Edgard Santos, Universidade Federal da Bahia, Salvador, BA, Brazil

Critical Care 2013, 17(Suppl 3):P35 (doi: 10.1186/cc12651)

Introduction Delirium assessment is already a well-established practice in the ICU. Usually, these evaluations are represented as delirium incidence or delirium-free days without coma. We propose four derived variables to predict the outcome in delirium patients in order to identify the most accurate data.

Methods A prospective study took place in the ICU of the University Hospital Professor Edgard Santos, Salvador, Brazil during the period of January to March 2013. Adult patients were assessed twice daily for detection of delirium with the Confusion Assessment Method for the ICU. Those patients with less than 48 hours in the ICU were excluded. The derived variables were classified into four groups: Group 1 - days of delirium, Group 2 - delirium episodes; Group 3 - maximum time of consecutive positive delirium; Group 4 - delirium density (days of consecutive positive delirium/days of delirium). These variables were compared with the outcome - mortality - of patients with positive delirium during the ICU stay. SPSS 21 for Windows was used for statistical analyses.

Results Forty-five patients were analyzed, 16 of whom presented delirium. The mortality of delirium patients was $60 \%$. Group 1: 1 day with delirium was associated with mortality of $60 \%$ compared with $50 \%$ with 2 days or more. Group 2: patients who had just one episode had $67 \%$ mortality compared with $50 \%$ mortality if they have two or more episodes. Group 3: mortality with a density $\geq 0.5$ was $57 \%$ versus $50 \%$ mortality in the subgroup $<0.5$. Finally, Group 4 : comparing the patients who died with the survival patients, we found 1.75 days of consecutive positive evaluation in the patients who died and 4 days in those who survived. None of the differences between these results for any of the four groups were statistically significant.

Conclusion Owing to the small population analyzed we could not conclude which was the best variable to predict delirium outcomes. None of the variables analyzed affected outcome when compared with just one positive evaluation during the ICU stay. New studies with a larger population are needed to identify the best variable.

\section{P36}

Pharmacological treatment of hypoactive delirium in critically ill patients: a systematic review

JPLM Carvalho', R Alvim¹, J Martins', C Bouza', P Zenaide², R Zantieff', B Pondé1, D Amorim', LC Quarantini', D Gusmao-Flores

'Faculdade de Medicina da Bahia, Universidade Federal da Bahia, Salvador, BA, Brazil: '2Escola Bahiana de Medicina e Saúde Pública, Salvador, BA, Brazil: ${ }^{3}$ University Hospital Prof. Edgard Santos, Universidade Federal da Bahia, Salvador, BA, Brazil

Critical Care 2013, 17(Suppl 3):P36 (doi: 10.1186/cc12652)

Introduction Delirium is generally managed by treating its underlying causes. However, symptomatic treatment may also be indicated. Although hypoactive delirium in critically ill patients is the most prevalent subtype of delirium, the effects of treatment with drugs specifically for this group are not well defined. The aim of this systematic review is to evaluate the role of pharmacological treatment in critically ill patients with hypoactive delirium.

Methods A systematic review was conducted, based on the PRISMA criteria, to identify articles on the pharmacological approach to hypoactive delirium in critically ill patients. First, a MEDLINE and SciELO database search was performed for articles published in the English language, involving patients in ICUs in which pharmacological therapy was used to treat delirium. Second, these studies were reevaluated to identify subtypes of delirium and the impact of the treatment.

Results The number of studies included in the qualitative synthesis was 18. One-half of them were clinical trials and the others were either letters or comments. However, only one study specified the treatment 
of hypoactive subtype delirium. The design of this study was a posthoc analysis of a double-blind, randomized, placebo-controlled study that used quetiapine as an adjuvant therapy to haloperidol. This study suggested that quetiapine appears to have more rapid resolution of many delirium symptoms, including a hypoactive state. These results were not statistically significant. The other 17 studies do not address the subtype.

Conclusion There is poor evidence regarding the use of drugs for the management of hypoactive delirium. Not only the study design but the number of patients studied in the single trial is very limited, which affects the power of evidence. Double-blind, randomized, placebo controlled trials must be performed to guide the treatment and the management of hypoactive delirium.

Acknowledgements JPLMC and RA contributed equally to this work. Reference

1. Devlin et al: Impact of quetiapine on resolution of individual delirium symptoms in critically ill patients with delirium: a post-hoc analysis of a double-blind, randomized, placebo-controlled study. Crit Care 2011, 15:R215.

\section{Nutrition/Metabolism}

P37

Effect of $\mathrm{N}$-acetylcysteine on serum thyroid hormone levels in nonthyroidal illness syndrome

J Vidart, SM Wajner, BD Schaan, AL Maia

Hospital de Clínicas de Porto Alegre, Santa Cecília, Porto Alegre, RS, Brazil

Critical Care 2013, 17(Suppl 3):P37 (doi: 10.1186/cc12653)

Introduction Nonthyroidal illness syndrome (NTIS) refers to changes in thyroid hormone levels affecting up to $75 \%$ of critically ill patients. Cytokines and oxidative stress have been implicated as causative factors, as they derange deiodinase reactions. Interestingly, the addition of $N$-acetylcysteine (NAC) in a cell model prevented the effect of IL- 6 on deiodinases, probably through a mechanism that restores catalytic activity of the enzyme [1]. NTIS is an independent marker of poor prognosis during myocardial infarction (MI) [2]. Here, we investigate whether NAC administration would prevent the decrease in serum thyroid hormone levels observed in patients with MI.

Methods This was a randomized, multicenter clinical trial. Patients with MI within 12 hours of evolution who underwent primary percutaneous coronary intervention were eligible. Patients were randomized to receive NAC $(1,200 \mathrm{mg}$, intravenous, every 12 hours) or placebo for 48 hours. Baseline characteristics, clinical history and serial blood samples for measurements of thyroid hormones were collected. Primary outcome was the variation of serum T3 levels. Statistical tests used included $x^{2}$ test analyses for proportions and Student's $t$ test analyses for means.

Results Sixty-seven patients were included. There were no differences between groups with respect to baseline clinical characteristics. When compared with baseline, the levels of serum T3 decreased in the placebo group at 12 hours of follow-up (98.6 vs. $86.8 \mu \mathrm{g} / \mathrm{dl}, P=0.001)$, as expected for the NTIS. Interestingly, the T3 descendent curve was

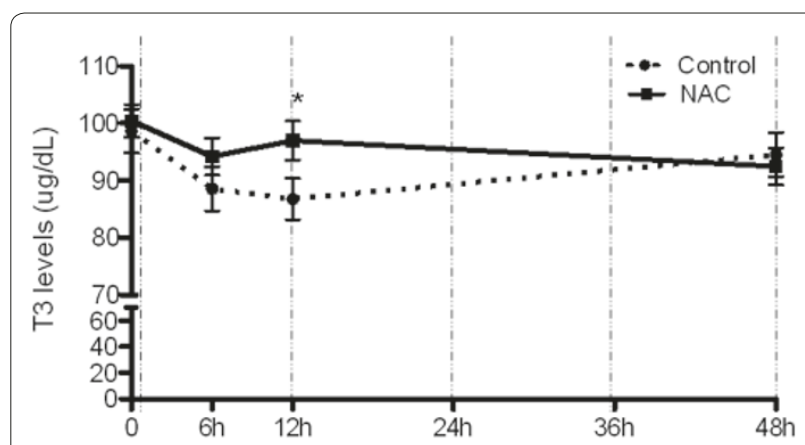

Figure 1 (abstract P37). attenuated in patients who were randomized to NAC (100.4 vs. $96.9 \mu \mathrm{g} /$ $\mathrm{dl}, P=0.396$ ) (Figure 1). Similar serum T3 levels were observed in both groups at 48 hours and on the 5th day. Serum TSH levels were virtually identical between the two groups. In the comparison between groups, the mean T3 serum levels were higher in the NAC treatment group at 12 hours than in the placebo group $(P=0.045)$.

Conclusion This is the first clinical trial designed to investigate the effect of NAC on NTIS in humans. We show that patients with myocardium infarction who received NAC treatment on admission had attenuation of the degree of $\mathrm{T} 3$ level decrease compared with those who received placebo. As expected, NAC did not interfere with central feedback, as TSH levels presented a similar rise in both groups, probably as a marker of recovery from acute illness. However, whether this effect is able to prevent mortality or to ameliorate patient outcome remains to be elucidated.

Acknowledgement Clinical Trials: NCT01501110.

References

1. Wajner SM, Goemann IM, Bueno AL, Larsen PR, Maia AL: J Clin Invest 2011, 121:1834-1845.

2. Wang F, Pan W, Wang S, Pan S, Ge J: Crit Care 2012, 16:R11.

\section{Pneumology}

P38

Association between use of lung-protective ventilation with lower tidal volumes and risk of acute lung injury, mortality, pulmonary infection, and atelectasis: a meta-analysis

VGM Pereira, A Serpa Neto, SO Cardoso, JA Manetta, DC Espósito, M de Oliveira Prado Pasqualucci, MJ Schultz

ABC Medical School (FMABC), Príncipe de Gales, Santo André, SP, Brazil Critical Care 2013, 17(Suppl 3):P38 (doi: 10.1186/cc12654)

Introduction Lung-protective mechanical ventilation with the use of lower tidal volumes has been found to improve the outcome of patients with acute lung injury (ALI) or its more severe form acute respiratory distress syndrome. It has been suggested that use of lower tidal volumes also benefits patients not suffering from ALI. The objective of this study was to test the hypothesis that use of lower tidal volumes is associated with improved outcomes of patients without ALI. Methods Data source A search of MEDLINE, CINAHL, Web of Science, and Cochrane Central Register of Controlled Trials up to August 2012. Study selection Eligible studies were those evaluating use of lower versus higher tidal volumes in patients without ALI at onset of mechanical ventilation and reporting lung injury development, overall mortality, pulmonary infection, atelectasis and biochemical alterations. Data extraction Three reviewers extracted data on study characteristics, methods, and outcomes. Disagreement was resolved by consensus.

Results Twenty articles (2,822 participants) were included. Metaanalysis using a fixed-effect model showed a decrease in lung injury development (risk ratio (RR), 0.33 ( $95 \% \mathrm{Cl} 0.23$ to 0.47 ); number needed to treat (NNT), 1 to 11), mortality (RR, 0.64 (95\% Cl 0.46 to 0.89 ); NNT, 1 to 23 ) and pulmonary infection (RR, 0.52 (95\% Cl 0.33 to 0.82 ); NNT, 1 to 26 ) in patients ventilated with lower tidal volumes. The results of lung injury development were similar when stratified by the type of study (randomized vs. nonrandomized), was significant only in randomized trials for pulmonary infection and only in nonrandomized trials for mortality. The hospital length of stay was lower in the protective ventilation group $(6.91 \pm 2.36$ vs. $8.87 \pm 2.93$ days, respectively; standardized mean difference, $0.60(95 \% \mathrm{Cl} 0.50$ to 0.71$))$. Protective ventilation was associated with higher PaCO levels (41.05 \pm 3.79 vs. $37.90 \pm 4.19 \mathrm{mmHg}$, respectively; SMD, $-0.47(95 \% \mathrm{Cl}-0.59$ to -0.34$)$ ), lower $\mathrm{pH}(7.37 \pm 0.03$ vs. $7.40 \pm 0.04$, respectively; SMD, $0.75(95 \% \mathrm{Cl}$ 0.58 to 0.92 )) but similar $\mathrm{PaO}_{2} / \mathrm{FiO}_{2}(304.40 \pm 65.7$ vs. $312.97 \pm 68.13$, respectively; SMD, 0.08 ( $95 \% \mathrm{Cl} 0.00$ to 0.16$)$ ). The tidal volume gradient between the two arms did not influence significantly the final results. Conclusion Among patients without ALI, protective ventilation with lower tidal volumes was associated with a better clinical outcome. Some of the limitations of our meta-analysis were the mixed setting of mechanical ventilation (ICU or operating room) and the duration of mechanical ventilation. 
P39

Comparison of CURB-65 and CRB-65 as predictors of death in community-acquired pneumonia in adults admitted to an ICU

AR Santana, FF Amorim, FB Soares, LG de Souza Godoy, L de Jesus Almeida, TA Rodrigues, GM de Andrade Filho, TA Silva, OG da Silva Neto, PHG Rocha, PN Ferreira Jr, APP Amorim, E Bastos de Moura, JA de Araújo Neto,

M de Oliveira Maia

Unidade de Terapia Intensiva Adulto do Hospital Santa Luzia, Asa Sul, Brasília, DF, Brazil

Critical Care 2013, 17(Suppl 3):P39 (doi: 10.1186/cc12655)

Introduction Community-acquired pneumonia is one of the most common causes of sepsis and ICU admissions. Patients with CAP who demand critical care had mortality rates of 25 to $50 \%$. Thereby, the assessment of the severity is essential to guide the treatment. There are several severity scores for CAP and some of the most acknowledged are CURB-65 and CRB-65. The objective of this study was to evaluate the accuracy of CURB- 65 and CRB- 65 as predictors of death in patients with community-acquired pneumonia.

Methods A prospective study during 6 months was conducted with patients diagnosed with CAP admitted to the ICU of the Hospital Santa Luzia, Brasília, DF, Brazil. Patients were stratified according to CURB-65 ( 0 to 5 ) and CRB-65 ( 0 to 4 ) and their risk categorized as: low (CURB-65: 0 to 1 and CRB-65: 0), moderate (CURB-65: 2 and CRB-65: 1 to 2) and high (CURB-65: 3 to 5 and CRB-65: 3 to 4). The sensitivity, specificity, positive predictive value (PPV), negative predictive value (NPV), likelihood ratio positive $(\mathrm{LR}+)$, and likelihood ratio negative (LR-) were calculated. Validity and reliability were assessed with the Spearman correlation coefficient. Patients with chronic kidney failure and those submitted to mechanical ventilation at the time of admission were excluded.

Results A total of 62 patients were included. Twenty-seven with low risk, 24 with moderate risk and 11 with high risk according to CURB65 and their mortality rates were $7.4 \%, 8.3 \%$ and $54.5 \%$, respectively. According to CRB-65, 11 were low risk, 44 moderate risk and seven had high risk. The mortality on CRB-65 stratification was $0 \%, 15.9 \%$ and $42.9 \%$ for low, moderate and high risks, respectively. When we gathered moderate and high risks, CRB- 65 was more sensitive (1.00 vs. $0.80)$ and had better LR- $(0.00$ vs. 0.41$)$, and NPV (1.00 vs. 0.92$)$. CURB-65 had better specificity (0.48 vs. 0.21$), \mathrm{LR}+(1.54 \mathrm{vs} .1 .26)$, and PPV ( 0.23 vs.

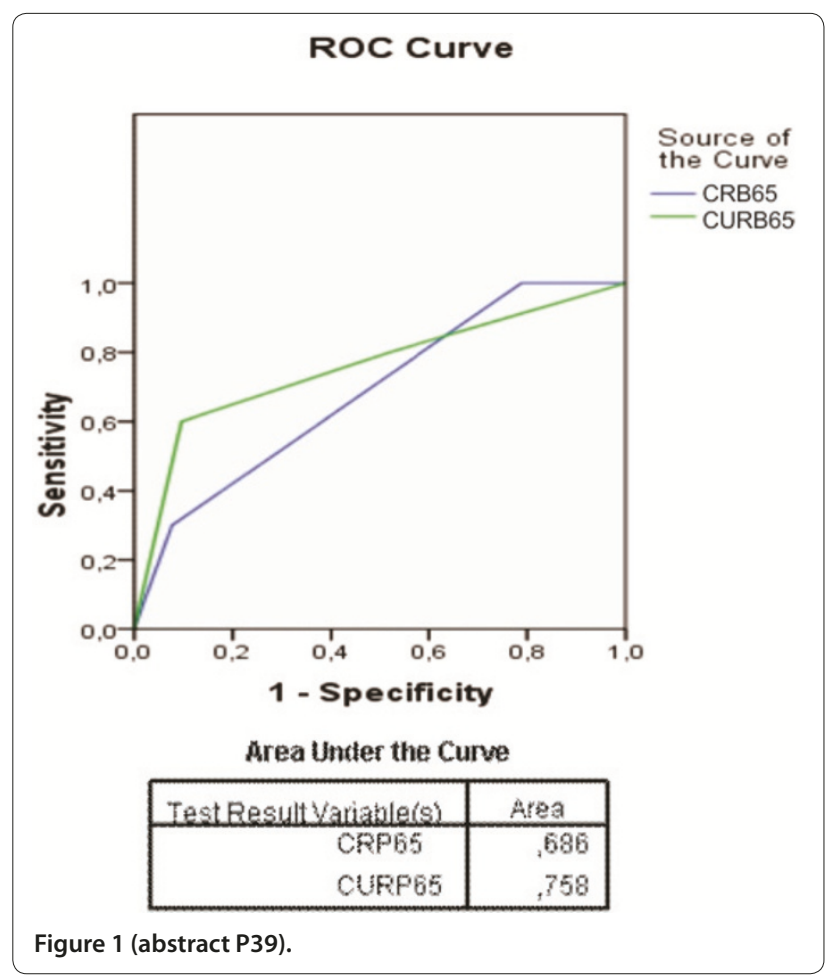

0.20). The receiver operating characteristic curves of CURB- 65 and CRB65 had areas of 0.758 and 0.686 , respectively. The Spearman correlation coefficient was $0.612(P=0.00)$. See Figure 1 .

Conclusion CURB-65 and CRB-65 had a high correlation. CRB-65 was more sensitive as a predictor of death as well as a guidance for hospitalization. Moreover, CRB-65 is a more practical score since it does not use laboratorial variables.

References

1. Ewig S, de Roux A, Bauer T, et al:: Validation of predictive rules and indices of severity for community acquired pneumonia. Thorax 2004, 59:421-427.

2. Correa RA, et al.: Brazilian guidelines for community-acquired pneumonia in immunocompetent adults - 2009. J Bras Pneumol 2009, 35:574-601.

P40

Influence of a humidification system on ventilator-associated

pneumonia: a randomized controlled trial

JRA de Azevedo, A da Luz Leitão, NNP Souza, MP Pereira

Hospital São Domingos, Bequimão, São Luis, MA, Brazil

Critical Care 2013, 17(Suppl 3):P40 (doi: 10.1186/cc12656)

Introduction Heat and moisture exchanger (HME) filters are replacing heated humidifiers $(\mathrm{HH})$ to promote humidification of inhaled air during mechanical ventilation. The argument is often that the HMEs are related to a lower incidence of VAP. On the other hand, a restriction to HMEs is that they are associated with an increased incidence of obstruction of the endotracheal tube. It is now known that the ventilator circuit and its accessories, including the humidification system, have very little impact on the incidence of VAP. The aspiration of contaminated secretions is the main determinant of this complication. The objective of this study was to compare the incidence of VAP in patients undergoing mechanical ventilation using $\mathrm{HH}$ versus HME filter. Secondarily, we analyzed the incidence of tracheal tube obstruction in both groups.

Methods This prospective randomized study included all adult patients undergoing mechanical ventilation for more than 12 hours, admitted to a 37-bed general ICU in the period from October 2011 to January 2013. The study was approved by the Ethics Committee of Hospital São Domingos under number 026/ 2011. Group 1 used HME filters TWINSTAR 55 (Drager Medical, Germany). The filter was changed every 24 hours. Group 2 used heated humidifier MR 730 (Fisher and Paykel Healthcare Inc., Auckland, New Zealand). The humidifier was exchanged between patients with the ventilator circuit.

Results One hundred and fifty-three patients were randomized. Two were excluded: excessive airway secretion forcing frequent exchanges of HME filter (G1); length of MV $<12$ hours (G2). One hundred and fifty-one patients were analyzed. The two groups were comparable with regard to demographic data and severity (APACHE IV and SOFA). The main indication of $\mathrm{MV}$ was hypoxemic respiratory failure $(\mathrm{G} 1=34 ; \mathrm{G} 2=35)$. The duration of mechanical ventilation was comparable between the two groups (G1 = $10.6 \pm 19.0$ days; $G 2=12.0 \pm 20.7$ days; $P=0.65$ ). The incidence of VAP also did not differ significantly between groups: $G 1=7(9.2 \%), G 2=10(13.3 \%)$; $P=0.42$. No endotracheal tube occlusion was identified.

Conclusion This study, like several others, including a meta-analysis of the Cochrane Institute (2,236 patients), failed to show that HME filters are able to reduce the incidence of ventilator associated pneumonia, compared with heated humidifiers. We had no cases of obstruction of the endotracheal tube. There is evidence in the literature that an HME filter change at intervals greater than that we used are related to a higher incidence of artificial airway occlusion. The choice of the system of humidification of inspired air in patients on mechanical ventilation (heated humidifier filter or HME) should not have the purpose to reduce the incidence of VAP.

P41

Neuromuscular blocking agents in patients with acute respiratory distress syndrome: a summary of the current evidence from three randomized controlled trials VGM Pereira

ABC Medical School (FMABC), Príncipe De Gales, Santo André, SP, Brazil Critical Care 2013, 17(Suppl 3):P41 (doi: 10.1186/cc12657)

Introduction Acute respiratory distress syndrome (ARDS) is a potentially fatal disease with high mortality. Our aim was to summarize 
the current evidence for use of neuromuscular blocking agents (NMBA) in the early phase of ARDS.

Methods A systematic review and meta-analysis of publications between 1966 and 2012. The Medline and CENTRAL databases were searched for studies on NMBA in patients with ARDS. The meta-analysis was limited to: randomized controlled trials; adult human patients with ARDS or acute lung injury; and use of any NMBA in one arm of the study compared with another arm without NMBA. The outcomes assessed were: overall mortality, ventilator-free days, time of mechanical ventilation, adverse events, and changes in gas exchange, in ventilator settings, and in respiratory mechanics.

Results Three randomized controlled trials covering 431 participants were included. Patients treated with NMBA showed less mortality (risk ratio, $0.71(95 \% \mathrm{Cl}, 0.55$ to 0.90$)$; number needed to treat, 1 to 7$)$, more ventilator-free days at day $28(P=0.020)$, higher $\mathrm{PaO}_{2}$ to $\mathrm{FiO}_{2}$ ratios $(P=0.004)$, and less barotraumas $(P=0.030)$. The incidence of critical illness neuromyopathy was similar $(P=0.540)$.

Conclusion The use of NMBA in the early phase of ARDS improves outcome.

\section{P42}

Profile of reintubated patients submitted to daily weaning screen and spontaneous breathing trial in a general ICU

NR Machado, CSM Silva, C Taniguchi, E Giovanetti, PA Pellegrini,

D Carineli-Cazati, GFJ Matos, RC Eid, KT Timenetsky

Hospital Israelita Albert Einstein, Morumbi, Sao Paulo, SP, Brazil

Critical Care 2013, 17(Suppl 3):P42 (doi: 10.1186/cc12658)

Introduction Mechanical ventilation (MV) weaning is the transition of artificial ventilation to spontaneous breathing of patients intubated for more than 24 hours. Reintubation may occur, even if the weaning process has been well conducted, in 13 to $19 \%$ of the extubated patients. Daily weaning screen and spontaneous breathing trial are widely used to evaluate patients ready to be weaned, although a reintubation risk may occur [1]. The objective of this study was to verify the profile of patients that failed the weaning process and needed to be reintubated.

Methods Mechanically ventilated patients submitted to our institutional MV weaning protocol from January to July 2012, who were extubated and failed extubation within a 48-hour period, were included in the study. The weaning protocol consisted of daily weaning screen and spontaneous breathing trial. Demographic data, MV time, ICU and hospital length of stay, causes of reintubation, and mortality rate were collected during the study period.

Results Two hundred patients were included, and 29 (14\%) were reintubated. Of the reintubated patients, $59 \%$ were male, with a median age of 69 years (range of 24 to 94), mean Simplified Acute Physiology Score (SAPS 3) of $60 \pm 11$, mean MV time of 9 days \pm 5 , median ICU stay of 14 days (range of 5 to 30), and 46 days of hospital stay. Causes of reintubation were acute respiratory failure (38\%), low level of consciousness associated with lack of airway protection (27\%), and hemodynamic instability (14\%). ICU discharge occurred in $70 \%$ of the patients, and 31\% were tracheostomized due to dysphagia, low level of consciousness, or lack of airway protection. The ICU mortality rate was $30 \%$. Only one tracheostomized patient died. Patients with ages ranging from 86 to 88 years had a higher incidence of low consciousness level. Patients that did not use noninvasive ventilation (NIV) after extubation were reintubated earlier than others (median of 20 hours, $P<0.02$ and $r=-0.551$ ), although there was no correlation with the use of NIV with mortality or MV time.

Conclusion The use of daily screening and spontaneous breathing trial is associated with a low reintubation rate. Acute respiratory failure and a low level of consciousness were the most common causes of reintubation, and most patients were discharged from the ICU. NIV may prevent the need for reintubation. Patients with no perspective of short-term improvement in level of consciousness may be considered for a tracheostomy.

Reference

1. Thille AW, Cortés-Puch I, Esteban A: Weaning from the ventilator and extubation in ICU. Curr Opin Crit Care 2013, 19:57-64.
P43

Spontaneous breathing trial reduces mechanical ventilation weaning when compared with SmartCare ${ }^{\mathrm{TM}}$ ventilation

CTaniguchi, KT Timenetsky, CSM Silva, E Giovanetti, R Henn, RAC Eid, CSV Barbas

Albert Einstein Jewish Hospital, Morumbi, São Paulo, SP, Brazil

Critical Care 2013, 17(Suppl 3):P43 (doi: 10.1186/cc12659)

Introduction Mechanical ventilation (MV) weaning is commonly performed using a spontaneous breathing trial (SBT) with pressure support ventilation after a daily weaning screen [1]. Recently there has been increased interest in automatic weaning trials, using respiratory rate, tidal volume and $\mathrm{ETCO}$, monitoring during SBT $[1,2]$. So far there is no clinical evidence comparing an automatic weaning trial with SBT. Our study's objective was to compare MV and weaning times between Automatic Weaning Ventilation System (SmartCare ${ }^{\text {TM }} / \mathrm{PS}$ ) and SBT groups.

Methods A randomized, controlled study was performed in a general ICU. We enrolled adult patients who were ventilated for more than 24 hours. Patients were randomized either to the control or SmartCare ${ }^{\mathrm{TM}}$ group. All patients were ventilated with a Drager EvitaXL (Drager Medical, Lubeck, Germany) ventilator with SmartCare ${ }^{\text {TM}} / \mathrm{PS}$ software version 1.1. The control group consisted of a daily weaning screen and SBT with pressure support ventilation; if patients tolerated SBT they were extubated. SmartCare ${ }^{\mathrm{TM}}$ group patients were also submitted to a daily weaning screen, after which they were ventilated with the SmartCare ${ }^{\mathrm{TM}} / \mathrm{PS}$ mode. We evaluated the MV and weaning time, maximum inspiratory pressure, maximum expiratory pressure, vital capacity, respiratory frequency to tidal volume ratio $(\mathrm{f} / \mathrm{Vt})$, use of noninvasive ventilation (NIV) post extubation, and re-intubation rate.

Results We evaluated a total of 70 patients (35 patients randomized in each group). There was no difference in age $(P=0.298)$ or gender $(P=0.08)$ between groups (Table 1). There was no difference in MV time between the control and SmartCare group $(P=0.534)$ (Table 1). Weaning duration was lower in the control group (pf $/ \mathrm{VT}, P=0.414$ ), use of NIV post extubation $(P=0.811)$ and re-intubation rate $(P=1.0)$ (Table 1$)$.

Table 1 (abstract P43). Characteristics of patients between SmartCare ${ }^{\mathrm{TM}}$ and control groups

\begin{tabular}{lccc}
\hline & SmartCare $^{\text {TM }}$ & Control & Pvalue $^{*}$ \\
\hline Idade & $60(46$ to 77$)$ & $65(57$ to 81$)$ & 0.298 \\
Gender, male & $23(65)$ & $16(45)$ & 0.07 \\
MIP & $45(40$ to 53$)$ & $40(36$ to 50$)$ & 0.272 \\
MEP & $40(30$ to 59$)$ & $40(21$ to 44$)$ & 0.059 \\
VC & $1,200(900$ to 1,850$)$ & $1,000(500$ to 1,600$)$ & 0.834 \\
f $N$ t & $35(24$ to 55$)$ & $40(26$ to 68$)$ & 0.414 \\
MV time & $4(2$ to 6$)$ & $3(2$ to 7$)$ & 0.534 \\
Weaning time & $110(80$ to 120$)$ & $60(50$ to 80$)$ & $<0.001$ \\
Use of NIV post extubation & $18(51.4)$ & $16(45.7)$ & 0.811 \\
Reintubation rate & $2(5.7)$ & $2(5.7)$ & 1.00 \\
\hline
\end{tabular}

Data presented as median (interquartile range) or $n$ (\%). f $/ \mathrm{VT}$, respiratory rate to tidal volume ratio; MEP, maximum expiratory pressure; MIP, maximum inspiratory pressure; MV, mechanical ventilation; NIV, noninvasive ventilation; VC, vital capacity. ${ }^{*} P$ value significant $<0.05$.

Conclusion SBT showed a reduction in weaning time when compared with the SmartCare ${ }^{\mathrm{T}} / \mathrm{PS}$ group, although there was no impact on total $\mathrm{MV}$ time and reintubation rate.

References

1. Blackwood B, Alderdice F, Burns K, et al.: Use of weaning protocols for reducing duration of mechanical ventilation in critically ill adult patients: Cochrane systematic review and meta-analysis. BMJ 2011, 342:C7237.

2. Lellouche $F$, Mancebo J, Jolliet $P$, et al:: A multicenter randomized trial of computer-driven protocoled weaning from mechanical ventilation. Am J Respir Crit Care Med 2006. 174:894-900. 
P44

Impact of acute brain dysfunction on the outcomes of mechanically ventilated cancer patients

JIF Salluh', ', ICT Almeida', M Soares ${ }^{1,2}$, FA Bozza ${ }^{2,3}$, CR Shinotsuka ${ }^{1,2}$,

R Bujokas', VC Souza-Dantas', EW Ely ${ }^{4,5}$

'Instituto Nacional de Câncer, Praça Cruz Vermelha, Rio De Janeiro, RJ,

Brazil; '2D'Or Institute for Research and Education, Rio de Janeiro, RJ, Brazil; ${ }^{3}$ Instituto de Pesquisa Evandro Chagas, IPEC, Fundação Oswaldo Cruz, Rio de Janeiro, RJ, Brazil; ${ }^{*}$ Vanderbilt University School of Medicine, Nashville, TN, USA: ${ }^{5}$ Veteran's Affairs Tennessee Valley Geriatric Research Education Clinical Center, Nashville, TN, USA

Critical Care 2013, 17(Suppl 3):P44 (doi: 10.1186/cc12660)

Introduction Delirium and coma are a frequent source of morbidity for ICU patients. Several factors are associated with the prognosis of mechanically ventilated (MV) cancer patients, but no studies have evaluated delirium and coma (acute brain dysfunction). The present study evaluated the frequency and impact of acute brain dysfunction on hospital mortality.

Methods The study was performed at the National Cancer Institute, Rio de Janeiro, Brazil. We prospectively enrolled patients ventilated $>48$ hours with a diagnosis of cancer. The presence of acute brain dysfunction was assessed daily during the first 14 days of ICU care using the CAM-ICU. Patients were followed until hospital discharge. Univariate and multivariate analysis were performed to evaluate factors associated with hospital mortality.

Results A total 170 patients were included. Seventy-three percent had solid tumors, age 65 (53 to 72 (median, IQR 25 to $75 \%$ )) years. Admission SAPS II score was 54 (46 to 63) points and SOFA score was (7 (6 to 9)) points. Median duration of MV was 13 (6 to 21) days and ICU stay was 14 (7.5 to 22 ) days. ICU mortality was $54 \%$ and hospital mortality was $66 \%$. Acute brain dysfunction was diagnosed in 161 patients (95\%). Nonsurvivors had a higher frequency of acute brain dysfunction (110 $(97.3 \%)$ vs. 51 (89.4\%), $P=0.06)$. Survivors had more delirium/coma-free days (4 (1.5 to 6) vs. 1 ( 0 to 2 ), $P=0.771$ ( 0.681 to 0.873 ), $P<0.001$ ).

Conclusion Acute brain dysfunction in MV cancer patients is frequent and independently predictive of increased hospital mortality. Future studies should investigate means of preventing or mitigating acute brain dysfunction as they may have a significant impact on clinical outcomes.

Acknowledgements Supported by the National Cancer Institute, CNPq and FAPERJ.

\section{Sepsis}

\section{P45}

Comparative analysis of survival between older and nonolder severe sepsis and septic shock resuscitated patients

H Palomba, TD Correa, MSC Assunção, A Pardini, DR de Melo, E Silva

Hospital Israelita Albert Einstein, Morumbi, São Paulo, SP, Brazil

Critical Care 2013, 17(Suppl 3):P45 (doi: 10.1186/cc12661)

Introduction Advanced age has been associated with increased mortality in severe sepsis and septic shock patients [1,2]. However, the impact of early resuscitation following the Surviving Sepsis Campaign Guidelines in this population of patients is unclear. The objective of this study was to compare the in-hospital mortality between older (EP) and nonolder (N-EP) resuscitated patients according to the Surviving Sepsis Campaign Guidelines.

Methods A retrospective observational study. All patients with severe sepsis and septic shock admitted to the ICU between January 2006 and March 2012 were studied. Comparisons were performed between older ( $\geq 65$ years) and nonolder patients ( $<65$ years).

Results A total of 913 patients with severe sepsis and septic shock were included in this analysis. Older patients accounted for $63 \%(573 / 913)$ of patients and nonolder patients for $37 \%$ (340/913) of patients. The median (IQR) age was 80 years ( 73 to 85 ) and 51 years (40 to 59) for EP and N-EP, respectively. The incidence of severe sepsis ( $43 \%$ vs. $44 \%$ ) and septic shock ( $57 \%$ vs. $56 \%$ ) did not differ between the EP and N-EP groups $(P=0.78)$. EP patients had a higher median (IQR) APACHE II score

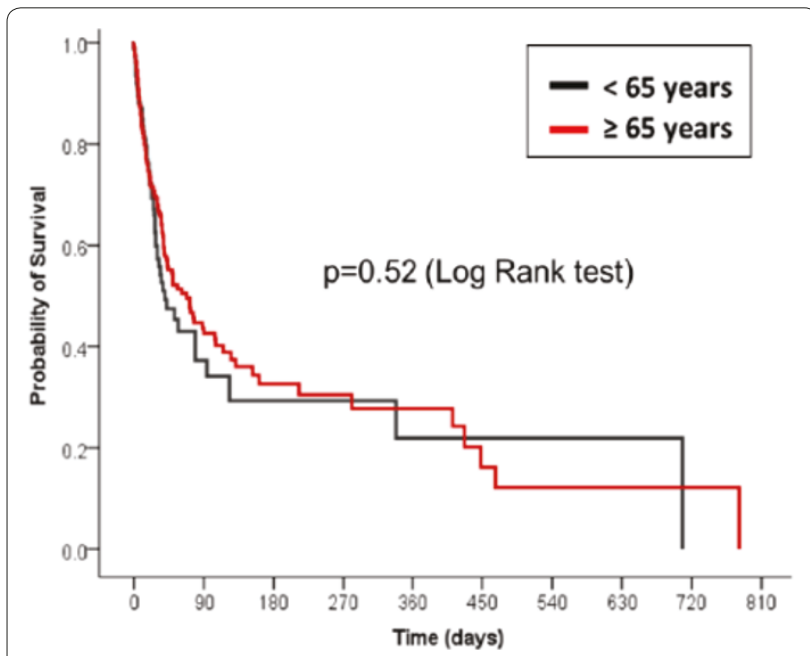

Figure 1 (abstract P45). Survival analysis comparing older and nonolder patients.

(23 (18 to 28$)$ ) than N-EP patients (19 (16 to 24$), P<0.001$ ), although the median number of organ dysfunctions ( 3 vs. 2 for EP and N-EP, respectively, $P=0.57$ ) did not differ between the groups. EP patients were more likely to have hypertension ( $51 \%$ vs. $29 \%, P<0.001)$, diabetes ( $33 \%$ vs. $24 \%, P=0.02)$, ischemic heart disease ( $16 \%$ vs. $7 \%, P<0.001)$ and chronic renal failure $(8.5 \%$ vs. $4.2 \%, P<0.03)$ when compared with N-EP patients. Solid organ transplantation $(24 \%$ vs. $4 \%, P<0.001)$ and liver cirrhosis $(17 \%$ vs. $5 \%, P<0.001)$ were more frequently in $\mathrm{N}$-EP patients. There was no significant between-group difference in the inhospital mortality (33\% in the EP group and $28 \%$ in the N-EP group; odds ratio, $1.27 ; 95 \% \mathrm{Cl}, 0.94$ to $1.70 ; P=0.12$ ) (Figure 1 ). The length of hospital stay (14 (7 to 29 ) vs. 12 (6 to 21 ) days (median (IQR)), $P=$ 0.001 ) was significantly higher in EP patients compared with the N-EP patients.

Conclusion In this population of severe sepsis and septic shock patients, the early resuscitation of older patients was not associated with increased mortality. However, prospective studies addressing the long-term impact of the resuscitation maneuvers on outcomes are necessary.

References

1. Dombrovskiy VY: Crit Care Med 2007, 35:1244-1250.

2. Martin GS: Crit Care Med 2006, 34:15-21.

P46

Effect of vasoactive drugs on the response of the baroreceptor and regulation of heart rate variability in patients with septic shock

AC Nogueira, V Kawabata, P Lotufo, L Ferandes, R Brandão, R Jenner,

C Mostarda, MC Yrigoyen, H Barbeiro, FG Soriano

Hospital Universitário da Usp, Butantã, São Paulo, SP, Brazil

Critical Care 2013, 17(Suppl 3):P46 (doi: 10.1186/cc12662)

Introduction We evaluated the action of vasoactive drugs on baroreceptor regulation and the interaction of this in regulation of heart rate variability (HRV) in patients with septic shock.

Methods A prospective observational study of patients with severe sepsis or septic shock. Collected data were analyzed retrospectively, separating patients according to their clinical evolution: survival or death. We monitored troponin I, vasoactive drug dose, HRV and blood pressure variability.

Results The study included 31 patients, of whom 14 died. Increased troponin levels were related to an increased risk of mortality. The alpha index of HRV low frequency and high frequency indicates that the interaction of baroreceptor in the regulation of heart rate variability with changes in blood pressure had a significant reduction in patients with septic shock and death. The use of dobutamine in patients with 


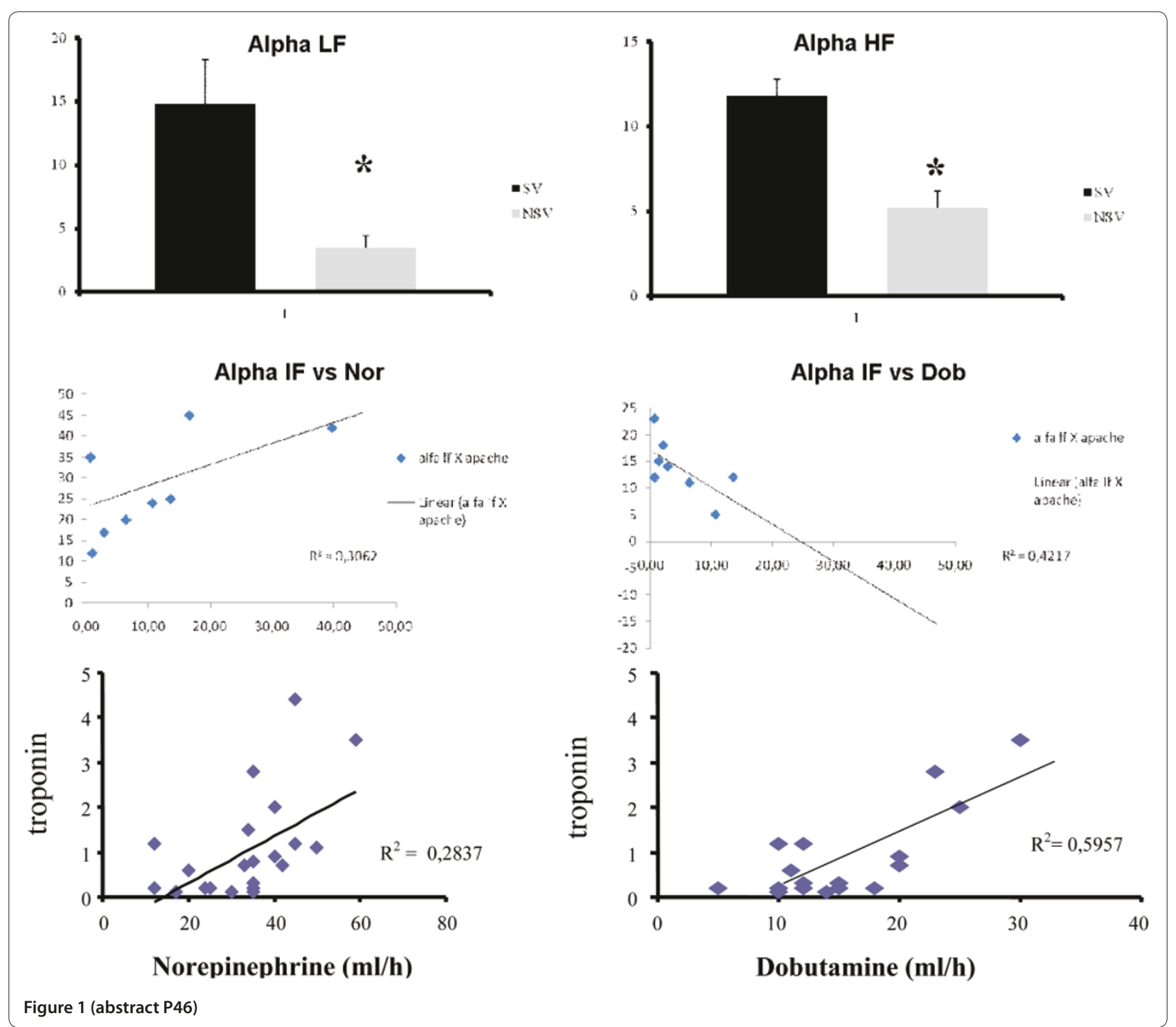

septic shock correlates well with troponin levels $(r=0.77)$, while for norepinephine the correlation was poor $(r=0.53)$. The use of dobutamine showed a negative correlation with the low-frequency alpha index (index that assesses the integration of baroreceptor); on the other hand, norepinephine showed a positive correlation. See Table 1 and Figure 1.

\section{Table 1 (abstract P46)}

\begin{tabular}{lc}
\hline Patients, $n$ & 30 \\
Gender, female & $12(40 \%)$ \\
Age (years) & $64 \pm 5$ \\
Nonsurvivors & $14(47 \%)$ \\
Infectious site & \\
$\quad$ Lung & $18(60 \%)$ \\
$\quad$ Abdominal tract & $5(15 \%)$ \\
$\quad$ Urinary tract & $4(14 \%)$ \\
$\quad$ Blood & $2(8 \%)$ \\
$\quad$ Nonidentified & $1(4 \%)$ \\
$\quad$ Gram-negative & $22(76 \%)$ \\
\hline
\end{tabular}

Conclusion Patients with septic shock have impaired baroreceptor function, and this is correlated with progression to death. Dobutamine is related to higher levels of cardiac damage and higher doses of dobutamine interfere with the responsiveness of the baroreceptor. Moreover, norepinephine has a positive effect on the integration of baroreceptor.

P47

Erythrocyte selenium concentration is a predictor of mortality in patients with septic shock

NA Costa, BLB Pereira, AL Gut, PS Azevedo, LAM Zornoff, SAR Paiva, MF Minicucci

Botucatu Medical School, UNESP, Rubião Júnior, Botucatu, SP, Brazil Critical Care 2013, 17(Suppl 3):P47 (doi: 10.1186/cc12663)

Introduction Severe sepsis and septic shock are major healthcare problems, affecting millions of people around the world each year, and increasing in incidence [1]. During sepsis there is increased oxidative stress, with reduced body stores of selenium (Se) and lower activity of glutathione peroxidase (GPX1), with patient outcomes of multiple organ failure and death [2]. The objective of this study was to determine the influence of Se concentration in plasma, erythrocytes and erythrocyte GPx1 activity on the length of hospital stay, length of ICU stay and ICU mortality in patients with septic shock. 
Methods This prospective study included all patients with septic shock on admission or during ICU stay, over the age of 18 , admitted to one of the three ICUs of the Botucatu Medical School, from January to August 2012. Demographic information, clinical evaluation and blood samples were taken within the first 72 hours of the patient's admission or within 72 hours after septic shock diagnosis for laboratory analysis, GPx activity, plasma and erythrocyte Se concentration. Categorical variables were analyzed by chi-squared or Fisher exact test. Continuous variables were analyzed by Student's $t$ test. For length of ICU or hospital stay, prediction multiple linear regression was used. For mortality prediction, multiple logistic regression was performed. The level of significance was set at $5 \%$. Results We evaluated 110 patients with a mean age of $58 \pm 16$ years and $63 \%$ were male. The median length of ICU stay and hospital stay was 9 (5 to 15$)$ and 18 (11 to 34$)$ days, respectively, and the ICU mortality rate was $55 \%$. Patients had an average plasma and erythrocyte Se concentration of $23.37 \pm 8.99$ and $32.83 \pm 11.89 \mathrm{~g} / \mathrm{l}$, respectively, and the median GPx1 activity was 30.56 (23.98 to 38.41 ) $\mathrm{U} / \mathrm{g} \mathrm{Hb}$. All patients had Se deficiency; however, only $25 \%$ had reduced GPx activity. There was no association of plasma and erythrocyte Se concentration and GPx activity with the length of hospital and ICU stay. Higher values of APACHE II, albumin and creatinine were associated with higher erythrocyte Se concentration. Regarding mortality, it was associated with higher lactate, urea, APACHE II and SOFA scores and lower values of albumin and length of hospital and ICU stay. In multiple logistic regression analysis adjusted for age, gender, and APACHE II, the erythrocyte Se concentration was a predictor of mortality in patients with septic shock (OR: $0.922 ; 95 \% \mathrm{Cl}: 0.880$ to $0.967 ; P<0.001$ ).

Conclusion The erythrocyte Se concentration is a predictor of mortality in patients with septic shock.

Acknowledgement Supported by CAPES.

\section{References}

1. Linde-Zwirble WT, Angus DC: Severe sepsis epidemiology: sampling, selection, and society. Crit Care 2004, 8:222-226.

2. Heyland DK, Dhaliwal R, Suchner U, Berger M: Antioxidant nutrients: a systematic review of vitamins and trace elements in the critically ill patient. Int Care Med 2005, 31:327-337.

P48

Evaluation of recognition and signalling receptors on the peripheral blood cells of septic patients and their correlation with clinical outcomes

SC Silva', GL Baggio-Zappia', MKC Brunialti', E Silva², LCP Azevedo³, FR Machado', R Salomao

'Hospital Sao Paulo, Escola Paulista de Medicina, Universidade Federal de Sao Paulo, SP, Brazil; ${ }^{2}$ Hospital Israelita Albert Einstein, Sao Paulo, SP, Brazil;

${ }^{3}$ Hospital Sirio Libanes, Sao Paulo, SP, Brazil

Critical Care 2013, 17(Suppl 3):P48 (doi: 10.1186/cc12664)

Introduction Monocytes and neutrophils play a key role in host defence by sensing and destroying microorganisms [1]. We evaluated the expression of cellular receptors implicated in pathogen recognition, cell activation and migration on both cell types during sepsis. Blood samples were collected from 77 septic patients (SP) at admission (D0), from 45 patients after 7 days of therapy (D7) and from 40 healthy volunteers (HV).

Results The expression of CD14 on monocytes and of CD11b and CXCR2 on neutrophils from SP was lower than that from HV. Conversely, the expression of TLR5 on monocytes and neutrophils was higher in SP when compared with HV. The expression of TLR2 on the surface of neutrophils and that of TLR5 on monocytes and neutrophils of SP was lower at D7 than at D0. In addition, the SP that survived showed reduced expression of TLR2 and TLR4 on the surface of neutrophils at D7 compared with D0 (Figure 1). The expression of CXCR2 for surviving patients was higher at follow-up when compared with baseline (Figure 1).

Conclusion The expression of recognition and cell signalling receptors is differentially regulated between SP and HV depending on the receptor being evaluated. However, despite these changes, it is likely that the functional changes in monocytes and neutrophils that are observed during sepsis are not directly linked to the modulation of the expression of TLRs [2].

References

1. Ley K: Molecular mechanisms of leukocyte recruitment in the inflammatory process. Cardiovasc Res 1996, 32:733-742.

2. Salomao R, Brunialti MK, Rapozo MM, Baggio-Zappia GL, Galanos C, Freudenberg M: Bacterial sensing, cell signaling, and modulation of the immune response during sepsis. Shock 2012, 38:227-242.

P49

Gene expression in peripheral mononuclear cells from septic patients secondary to community-acquired pneumonia: patterns of gene expression and outcomes

P Severino, E Silva, GL Baggio-Zappia, MKC Brunialti, O Rigato Jr,

IDC Guerreiro da Silva, FR Machado, R Salomao

Hospital Israelita Albert Einstein, Morumbi, São Paulo, SP, Brazil

Critical Care 2013, 17(Suppl 3):P49 (doi: 10.1186/cc12665)

Introduction Sepsis is defined as a systemic inflammatory response secondary to a proven or suspected infection. Mechanisms governing this inflammatory response have been shown to be complex and dynamic, involving cross-talk among diverse signaling pathways. However, current knowledge on mechanisms underlying sepsis is far from providing a complete picture of the syndrome, justifying additional efforts that might add to this scenario. Microarray-based expression profiling is a powerful approach for the investigation of complex clinical conditions such as sepsis: the analysis of gene transcription at the genome level potentially avoids results derived from biased assumptions. In this study we investigate whole-genome
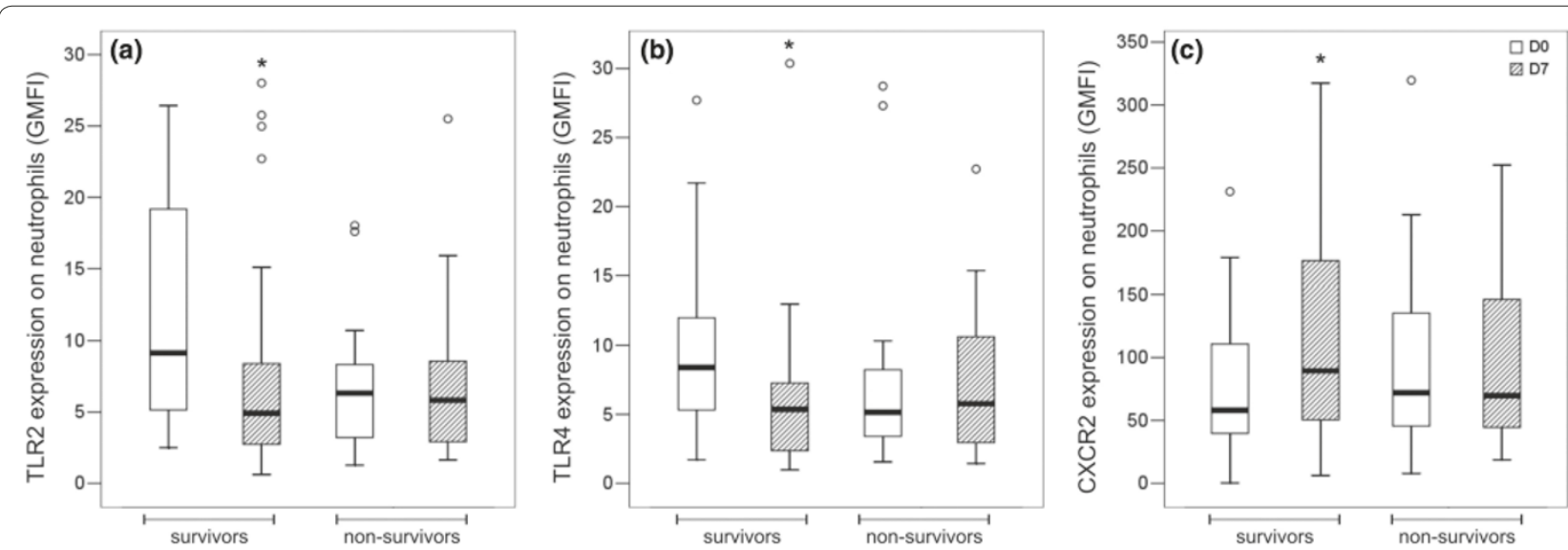

Figure 1 (abstract P48). Analysis of the expression of TLR2 (A), TLR4 (B) and CXCR2 (C) surface receptors on neutrophils from the whole blood of surviving septic patients and nonsurvivors at D0 and D7. Histograms and expressed as the geometric mean fluorescence intensity (GMFI). ${ }^{*} P<0.05$. 
gene expression profiles of mononuclear cells from survivor and nonsurvivor septic patients.

Methods Blood samples were collected at the time of sepsis diagnosis and 7 days later, allowing us to evaluate the role of biological processes or genes possibly involved in patient recovery. Aiming to circumvent, at least partially, the heterogeneity of septic patients, we included only patients admitted with sepsis caused by community-acquired pneumonia. Global gene expression profiling allowed us to characterize early sepsis, as compared with healthy individuals.

Results Our results corroborate literature reports on inflammation response in the early stages of sepsis but highlight great heterogeneity in gene expression during the onset of sepsis. Differences in oxidative stress seem to be associated with clinical outcome, since significant differences in the expression profile of related genes were observed between survivors and nonsurvivors. However, our results substantiate current knowledge supporting that sepsis syndrome development is indeed multifaceted. Although the initial infection of enrolled patients was pneumonia, with no sign of organ failure at the time of diagnosis, 7 days later gene expression profiles seemed to be characteristic for each patient, with no clear pattern of development. This result could be associated with the underlying health status of each one of them, with complications due to sepsis itself as well as with distinct timing for response to treatment.

Conclusion At this point we conclude that studies should focus on studying sepsis at multiple time points, aiming to capture common, although possibly separated in time when patients are considered, biological processes associated with recovery.

\section{P50}

Georeferencing of sepsis in São Paulo

F Colombari, D Diament, AS Cypriano, LF Lisboa, BF Cardoso dos Santos, MC Neto, E Silva

Hospital Israelita Albert Einstein, Morumbi, São Paulo, SP, Brazil

Critical Care 2013, 17(Suppl 3):P50 (doi: 10.1186/cc12666)

Introduction Morbidity and lethality in sepsis varies according to demographic and socioeconomic factors, which can be linked to geographic distribution of populations. The objective of this study is to relate sepsis deaths with geographic distribution in the city of São Paulo, Brazil.

Methods Death certificates for sepsis and sepsis-related infections (pneumonia, urinary tract infections, meningitis, skin and soft tissue infections, peritonitis, multiple organ failure) from 2004 to 2009 in the city of São Paulo, Brazil, were searched. Patients were distributed according to sex, age, main cause of death, secondary cause of death, residence address, and death location. The Human Development Index (HDI) was used to compare city districts. Health institutions were identified as public or private administered according to Ministry of Health registration.

Results The number of deaths increased with age, but there is no difference between sexes for the whole population studied. However, in younger age groups (up to 18 years and from 18 to 64 years) deaths were more frequent for males than females $(53.9 \times 46.1 \%$ and $61 \times 39 \%$, respectively). In the age group older than 65 years there were $46.3 \%$ of deaths in males and $53.7 \%$ for females. Deaths occurred homogeneously in all city districts, but its frequency was larger in districts with higher HDI. Death location was $93.73 \%$ in hospitals and $5.47 \%$ in dwellings. From the identifiable institutions where deaths occurred, $52.4 \%$ were public, $46.4 \%$ were private and $1.2 \%$ were nonidentifiable. The mean death charge was $79 \%$ higher in public institutions compared with private ones. There was no visible difference in death distribution according to age and residence address. Comparing patients' residence address with death location showed a concentration near the closest hospital, without difference between private or public hospitals. There was a higher proportion of deaths for the age group older than 65 years in private hospitals. For the age group between 19 and 64 years, the proportion of deaths was slightly higher in public hospitals. The main cause of death was pneumonia, followed by sepsis, multiple organ failure, intra-abdominal infections, meningitis, skin and soft tissue infections and urinary tract infections. Distributing main death causes by age groups, patients older than 65 years died more over pneumonia, sepsis and multiple organ failure. Patients up to 18 years died more over urinary tract infections and meningitis. The age group between 19 and 64 years was distributed fairly amongst all death causes. Conclusion Georeferencing is a potent tool for epidemiological studies. Deaths occurred homogeneously in all city districts, but the frequency was larger in districts with higher HDI. As expected, older patients died in greater numbers, affected by respiratory tract infections, sepsis and multiple organ failure. Deaths occurred mainly in the public health system.

\section{P51}

Lactate as a prognostic marker in patients with severe sepsis or septic shock admitted to the ICU

LL Rocha, CMS Pessoa, G Colombo, TD Corrêa, MSC de Assunção

Hospital Israelita Albert Einstein, Morumbi, São Paulo, SP, Brazil

Critical Care 2013, 17(Suppl 3):P51 (doi: 10.1186/cc12667)

Introduction Severe sepsis and septic shock are conditions associated with high morbimortality worldwide despite improvements in its management in the last two decades. It is of great interest for intensive care physicians to have the opportunity to use biomarkers for prediction of severity of disease and prognostication in a way that the more severely ill the patient, the more aggressive the treatment should be. Lactate is globally available benchside. The presence of elevated lactate levels are associated with increased mortality in distinct critically ill patient populations. Although lactate levels above $4 \mathrm{mmol} / \mathrm{l}$ are the classical trigger for early-goal directed therapy, there are some studies showing that even normal or slightly elevated lactate levels are associated with worse outcome. The objective of this study is to evaluate stratified lactate serum levels at admission as a predictor of 28-day mortality.

Methods A retrospective cohort study where data were collected from electronic charts of adult patients diagnosed with severe sepsis or septic shock admitted to our ICU between July 2005 and December 2010. Data collected included the following: social and demographic, presence of organic dysfunction at admission, initial lactate level, APACHE II score, compliance with the institutional sepsis protocol, use of mechanical ventilation and 28-day mortality. Lactate levels were expressed in $\mathrm{mg} / \mathrm{dl}$ and stratified into four quartiles: (1) normal, <14.4 mg/dl; (2) mild elevated, 14.5 to $28 \mathrm{mg} / \mathrm{dl}$; (3) intermediate elevated, 28.1 to $36 \mathrm{mg} / \mathrm{dl}$; and (4) high elevated, $>36 \mathrm{mg} / \mathrm{dl}$. The study protocol was approved by the local ethics committee. Categorical variables were expressed as frequencies and/or percentages and continuous as mean \pm standard deviation. For proportions and means comparisons, the chi-square and Student $t$ tests were used, respectively. Statistical significance was set as $P<0.05$. A univariate analysis was performed and statistically significant variables were included in a logistic regression model. The SPSS $^{\circledR}$ software (IBM Corporation, USA) was used to perform the tests. Results A total of 760 patients were included, with mean age of $67.2 \pm 18.66$ years and $57.9 \%$ male. Mean APACHE II score was 21 and $46.4 \%$ were in mechanical ventilation and $64.7 \%$ were using vasopressors. Global mortality was $36.7 \%$. An intermediate compliance with the institutional sepsis protocol was observed. The best stratum to predict mortality in our study was the high elevated lactate level group $(P<0.0001)$.

Conclusion Lactate levels are one of the most used biomarkers in sepsis. When their level is superior to $36 \mathrm{mg} / \mathrm{dl}$ patients are at highest risk of mortality and an aggressive resuscitation strategy shall be warranted in these patients.

\section{P52}

Procalcitonin as a prognostic biomarker of severe sepsis and septic shock

JRA de Azevedo, OJM Torres, O Malafaia

Hospital São Domingos, Bequimão, São Luis, MA, Brazil

Critical Care 2013, 17(Suppl 3):P52 (doi: 10.1186/cc12668)

Introduction Procalcitonin (PCT), the precursor peptide of calcitonin, has extremely low levels in healthy subjects. In response to bacterial infectious stimulation, PCT serum levels rise substantially. Recently PCT has been used as a biomarker for prognosis of severe sepsis and septic shock. Some studies have shown that isolated high levels do not predict outcome. Encouraging results were obtained with the 
evaluation of serum PCT levels. The objective of this study was to evaluate the tendency of the plasma concentration and clearance of PCT as biomarkers for prognosis of patients with severe sepsis and septic shock, compared with another early prognosis marker, the number of SIRS criteria at sepsis diagnosis.

Methods We conducted a prospective, observational, cohort study, with patients with severe sepsis and septic shock. The serum procalcitonin was determined at diagnosis of sepsis and after 24 and 48 hours. Demographic data, APACHE IV score, SOFA score on arrival, number of SIRS criteria at diagnosis, site of infection and microbiological results were recorded.

Results Twenty-eight patients were included, 19 clinical and nine surgical. In 13 patients (46.4\%) the source of sepsis was pulmonary, abdominal in seven cases (25.0\%), urinary in five cases $(17.9 \%)$ and soft tissue in three cases (10.7\%). Fifteen patients had severe sepsis and 13 septic shock. Overall mortality was $17.9 \%$ (five patients), three with septic shock. Twenty-eight PCT determinations were performed at sepsis diagnosis, 27 after 24 hours and 26 after 48 hours. The initial concentration was not significantly different between the survivor and nonsurvivor groups, but the differences between the two groups after 24 and 48 hours were statistically significant. There was no difference in the number of SIRS criteria. The 24-hour procalcitonin clearance proved to be significantly higher in the group of survivors $(-3.0$ vs. -300.0 , $P=0.028$ ). Although the 48-hour procalcitonin clearance was shown to be higher in the group of survivors when compared with nonsurvivors, the difference did not reach statistical significance.

Conclusion Persistently high PCT concentrations in plasma, as well as reduced 24-hour PCT clearance, were associated with a significant increase in mortality in patients with severe sepsis and septic shock.

P53

Serum C-reactive protein concentration in early abdominal and pulmonary sepsis

JA Orati', SM Lobo

'University of Ribeirão Preto, SP, Brazil; ${ }^{2 H o s p i t a l}$ de Base de São José do Rio

Preto, SP, Brazil

Critical Care 2013, 17(Suppl 3):P53 (doi: 10.1186/cc12669)

Introduction The objective of this study was to evaluate the C-reactive protein serum levels in patients with pulmonary and abdominal sepsis during the first 5 days of sepsis progression.

Methods The present investigation was a retrospective cohort study conducted at the university hospital with 345 patients who were admitted to the ICU and diagnosed with sepsis of pulmonary or abdominal origin. Serum C-reactive protein concentrations were measured by the turbidimetric immunoassay. For analysis of $\mathrm{C}$-reactive protein, day 1 was defined as the day on which the patient was clinically diagnosed with sepsis.

Results Thirty-four patients with sepsis (9.8\%), 114 patients with severe sepsis (33.0\%), and 197 patients with septic shock (57.2\%) were evaluated. The age of the patients was $56.4 \pm 19.8$ years. The serum C-reactive protein concentrations were higher on the day of sepsis diagnosis in the group with abdominal infection compared with the group with pulmonary sepsis $(17.8 \pm 10.1 \mathrm{mg} / \mathrm{dl}$ vs. $14.9 \pm 11.1 \mathrm{mg} /$ $\mathrm{dl}, P=0.025)$ and remained significantly higher during the first 5 days of sepsis progression. The values of the area under the ROC curve, sensitivity, specificity, and best cutoff points are listed in Table 1.

Table 1 (abstract P53). Values of the area under the ROC curve, sensitivity, specificity, and best cutoff points

\begin{tabular}{lcccc}
\hline ROC curve & $\begin{array}{c}\text { AUC } \\
(\mathbf{9 5 \%} \text { Cl) }\end{array}$ & Sensitivity & Specificity & $\begin{array}{c}\text { Cutoff } \\
\text { (mg/dl) }\end{array}$ \\
\hline CRP day 1 & $0.53(0.43$ to 0.62$)$ & 0.26 & 0.88 & 6.12 \\
CRP day 2 & $0.51(0.41$ to 0.60$)$ & 0.67 & 0.42 & 18.6 \\
CRP day 3 & $0.52(0.43$ to 0.61$)$ & 0.57 & 0.56 & 13.6 \\
CRP day 4 & $0.51(0.41$ to 0.60$)$ & 0.42 & 0.65 & 14.2 \\
CRP day 5 & $0.59(0.48$ to 0.68$)$ & 0.58 & 0.62 & 9.10 \\
\hline
\end{tabular}

AUC, area under the ROC curve; $\mathrm{Cl}$, confidence interval; CRP, C-reactive protein; $\mathrm{ROC}$, receiver operator characteristic.
Conclusion The accuracy of C-reactive protein for the differential diagnosis of pulmonary and abdominal sepsis is limited, although significantly higher serum levels were observed in patients with abdominal sepsis.

P54

Serum pro-metalloproteinase 9 is a predictor of length of ICU and hospital stay in patients with septic shock

NA Costa, AF Gonçalves, BPM Rafacho, AL Gut, PS Azevedo, LAM Zornoff, SAR Paiva, MF Minicucci

Botucatu Medical School, UNESP, Rubião Júnior, Botucatu, SP, Brazil

Critical Care 2013, 17(Suppl 3):P54 (doi: 10.1186/cc12670)

Introduction The matrix metalloproteinases (MMPs) participate in fundamental processes, such as cell proliferation, differentiation, adhesion, migration, angiogenesis, apoptosis, and inflammation [1]. The increased expression of MMPs suggests that these proteases may influence the pathogenesis of endotoxemia in sepsis [2]. The objective of this study was to evaluate the serum activity of MMP-2 and MMP-9, length of hospital stay, length of ICU stay and mortality in patients with septic shock.

Methods This prospective study included all patients with septic shock on admission or during ICU stay, over the age of 18 years, admitted to the ICU from March to July 2012. Demographic information, clinical evaluation and blood samples were taken within the first 72 hours of the patient's admission or within 72 hours after septic shock diagnosis for laboratory analysis and MMP-2 and MMP-9 activity. The activity of MMPs was performed by zymography. The level of significance was set at $5 \%$.

Results We evaluated 67 patients with a mean age of $56 \pm 15$ years, $66 \%$ male, the median length of ICU and hospital stay was 9 (4 to 15) and 16 (10 to 29 ) days, respectively, and the ICU mortality rate was $61 \%$. Higher values of APACHE II, SOFA, lactate and urea, and lower values of albumin, length of ICU and hospital stay were associated with ICU mortality. In univariate analysis, serum activity of MMP-2 and MMP-9 were not associated with length of ICU, hospital stay and mortality in septic shock patients. However, in the regression model analysis when adjusted for sex, age, lactate and APACHE II, the activity of pro-MMP-9 was negatively associated with the length of ICU (coefficient: -0.016 ; $P=0.028$ ) and hospital (coefficient: $-0.015 ; P=0.041$ ) stay, but was not associated with ICU mortality (OR: $1.051 ; 95 \% \mathrm{Cl}: 0.950$ to 1.163 ; $P=0.332$ ).

Conclusion Serum activity of pro-MMP-9 was negatively associated with length of ICU and hospital stay in patients with septic shock.

Acknowledgement Supported by CAPES.

References

1. Le NT, Xue M, Castelnoble LA, Jackson CJ: The dual personalities of matrix metalloproteinases in inflammation. Front Biosci 2007, 12:1475-1487.

2. Vanlaere I, Libert C: Matrix metalloproteinases as drug targets in infections caused by gram-negative bacteria and in septic shock. Clin Microbiol Rev 2009, 22:224.

P55

Serum thiamin concentration is negatively correlated with lactate levels in survivors of septic shock

NA Costa, MS Dorna, AL Gut, PS Azevedo, LAM Zornoff, SAR Paiva, MF Minicucci

Botucatu Medical School, UNESP, Rubião Júnior, Botucatu, SP, Brazil

Critical Care 2013, 17(Suppl 3):P55 (doi: 10.1186/cc12671)

Introduction Thiamine deficiency can be present in $20 \%$ of patients in the ICU. This deficiency is considered to be an uncommon source of lactic acidosis in septic patients. An elevated serum lactate level is associated with morbidity and mortality [1]. Increased levels of thiamine increase the activity of glutathione peroxidase (GPx), a major component of the cellular antioxidant system [2]. The objective of this study was to determine the influence of serum thiamine concentrations on lactate levels, GPx activity, length of hospital stay, length of ICU stay and ICU mortality in patients with septic shock.

Methods This prospective study included all patients with septic shock on admission or during ICU stay, over the age of 18 , admitted to one of the three ICUs of the Botucatu Medical School from January to August 
2012. Demographic information, clinical evaluation and blood samples were taken within the first 72 hours of the patient's admission or within 72 hours after septic shock diagnosis for laboratory analysis, serum thiamine and GPx activity determination. The level of significance was set at $5 \%$.

Results One hundred and eight consecutive patients were evaluated. The mean age was $57.5 \pm 16.0$ years, $63 \%$ were male and $54.6 \%$ died in the ICU. The frequency of thiamine deficiency was $71.3 \%$. Neither the serum thiamine concentration nor the erythrocyte GPx activity was associated with mortality in septic shock patients. Thiamine levels were also not associated with GPx activity $(r=0.141, P=0.165)$. In addition, thiamine levels were not associated with serum lactate in the 108 patients with septic shock $(r=-0.074, P=0.444)$. However, vitamin B1 levels were negatively associated with lactate in patients who survived $(r=-0.311, P=0.029)$. In the regression model analysis, vitamin B1 levels were not associated with ICU mortality or with the length of the ICU or hospital stay.

Conclusion Thiamine deficiency is common in septic shock patients. Furthermore, thiamine was negatively associated with lactate levels in survivors of septic shock.

Acknowledgement Supported by CAPES.

References

1. Wacharasint $P$, Nakada $T A$, Boyd JH, et al: Normal-range blood lactate concentration in septic shock is prognostic and predictive. Shock 2012, 38:4-10.

2. Gioda CR, de Oliveira Barreto T, Prímola-Gomes TN, et al.: Cardiac oxidative stress is involved in heart failure induced by thiamine deprivation in rats. Am J Physiol Heart Circ Physiol 2010, 298:H2039-H2045.

P56

Tissular perfusion influence on central, mixed and atrial venous oxygen saturations

SH Goto', BF Mazza², FR Machado²

${ }^{1}$ Hospital São Paulo, Universidade Federal de São Paulo, SP, Brazil; ${ }^{2}$ University

of São Paulo, Universidade Federal de São Paulo, SP, Brazil

Critical Care 2013, 17(Suppl 3):P56 (doi: 10.1186/cc12672)

Introduction Even though there has been quite a discussion on whether venous oxygen saturations are useful to guide treatment during initial resuscitation of sepsis, using mixed and central venous oxygen saturations as goals is still advised in the Surviving Sepsis Campaign under strong recommendation but a low level of evidence (1C). According to these guidelines, $\mathrm{SvO}_{2}<65 \%$ or $\mathrm{SvcO}_{2}<70 \%$ demands treatment. In addition, there is no consensus whether these variables are interchangeable. The objective of this study was to evaluate the influence of tissular perfusion on the correlation between the central venous $\left(\mathrm{SvCO}_{2}\right)$, the mixed venous $\left(\mathrm{SvO}_{2}\right)$ and the atrial oxygen saturations $\left(\mathrm{SvaO}_{2}\right)$ by the analysis of arterial lactate.

Table 1 (abstract P56). Baseline

\begin{tabular}{|c|c|c|c|}
\hline Variable & $\begin{array}{l}\text { Group } 1 \\
(n=37)\end{array}$ & $\begin{array}{l}\text { Group } 2 \\
(n=28)\end{array}$ & $P$ value \\
\hline Age & 66 (54 to 69) & 65 (54 to 69) & 0.171 \\
\hline Male (\%) & $35.1(13)$ & $85.7(24)$ & 0.000 \\
\hline APACHE ॥ & 18 (17 to 22) & 24 (20 to 29) & 0.016 \\
\hline SOFA admission & $6(5$ to 10$)$ & 10.5 (7 to 12.75$)$ & 0.007 \\
\hline SOFA sample & 9 (7 to 11$)$ & 11 (9.25 to 16$)$ & 0.007 \\
\hline $\mathrm{SVO}_{2}(\%)$ & 72 (68.5 to 76.5 ) & 70.5 (67 to 73 ) & 0.182 \\
\hline $\mathrm{SvCO}_{2}(\%)$ & 81 (76 to 85$)$ & 77 (72 to 80) & 0.016 \\
\hline $\mathrm{SvaO}_{2}(\%)$ & $77.9 \pm 8.6$ & $74.3 \pm 9.7$ & 0.274 \\
\hline $\mathrm{SaO}_{2}(\%)$ & 98 (97 to 98.5) & 95 (93 to 96) & 0.000 \\
\hline
\end{tabular}

Results expressed as \% ( $n$ ) or mean \pm standard deviation or median (25 to $75 \%$ percentiles). APACHE, Acute Physiologic Chronic Health Evaluation; SOFA, Sequential Organ Failure Assessment.
Table 2 (abstract P56). Spearman correlation $(r)$

\begin{tabular}{lccc}
\hline Variable & $\begin{array}{c}\text { Total } \\
(\boldsymbol{n}=65)\end{array}$ & $\begin{array}{c}\text { Group 1 } \\
(\boldsymbol{n}=37)\end{array}$ & $\begin{array}{c}\text { Group 2 } \\
(\boldsymbol{n}=28)\end{array}$ \\
\hline $\mathrm{SvO}_{2} \times \mathrm{SvCO}_{2}$ & $0.74^{*}$ & $0.66^{*}$ & $0.83^{*}$ \\
$\mathrm{SvO}_{2} \times \mathrm{SvaO}_{2}$ & $0.68^{*}$ & $0.60^{*}$ & $0.82^{*}$ \\
$\mathrm{SvCO}_{2} \times \mathrm{SvaO}_{2}$ & $0.72^{*}$ & $0.63^{*}$ & $0.85^{*}$ \\
\hline
\end{tabular}

*P $P<0.05$.

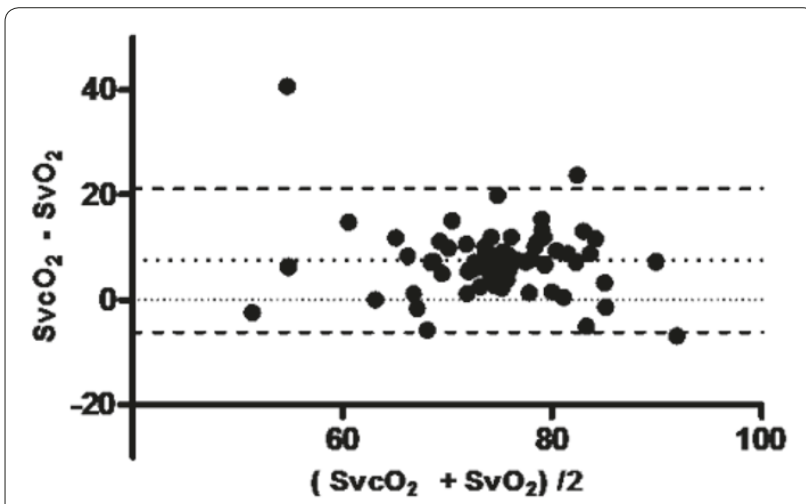

Figure 1 (abstract P56). Bland-Altman $\left(\mathrm{SVO}_{2} \times \mathrm{SvCO}_{2}\right)$ : bias 7.52, bias SD 6.957. 95\% Limits of agreement: -6.116 to 21.16 .

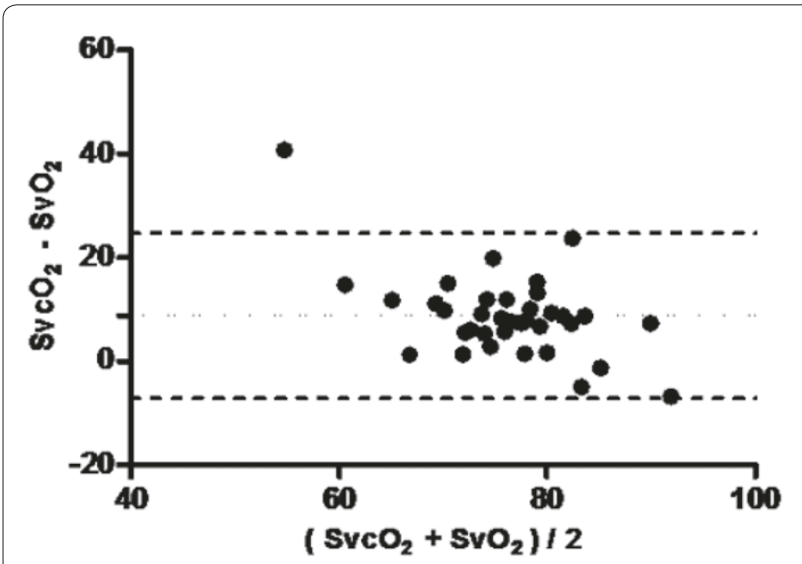

Figure 2 (abstract P56). Bland-Altman $\left(\mathrm{SvO}_{2} \times \mathrm{SvCO}_{2}\right)$ in Group 1: bias 8.778, bias SD 8.137. 95\% Limits of agreement: -7.171 to 24.73 .

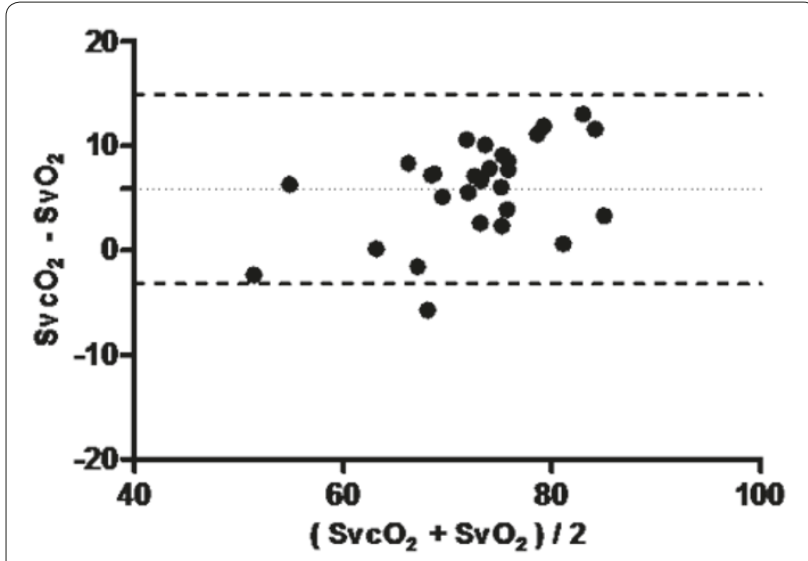

Figure 3 (abstract P56). Bland-Altman $\left(\mathrm{SvO}_{2} \times \mathrm{SvCO}_{2}\right)$ in Group 2: bias 5.857, bias SD 4.627. 95\% Limits of agreement: -3.211 to 14.93 . 
Methods A prospective observational study; the populations from three ICUs of the Hospital São Paulo were evaluated from October 2011 to November 2012 and patients diagnosed with severe sepsis or septic shock monitored by pulmonary artery catheter (PAC) were included. Hyperlactatemia was defined as an arterial lactate value $>28 \mathrm{mg} / \mathrm{dl}$ and the correct location of the PAC was confirmed by chest radiography and pulmonary artery pressure tracings. For the statistical analysis, samples were allocated into two groups: normal lactate levels (Group 1) and hyperlactatemia (Group 2). Results were expressed in mean \pm standard deviation or median ( 25 to $75 \%$ percentiles) or percentages.

Results Twenty-one patients were included; altogether, 65 paired blood samples were obtained (Table 1). A higher correlation between the venous oxygen saturations was found in the hyperlactatemia group (Table 2). APACHE II and SOFA scores were higher among these individuals (Table 1). $\mathrm{SvcO}_{2}$ and $\mathrm{SvO}_{2}$ were shown not to be acceptable surrogates by the analysis of the Bland-Altman plots, but bias and limits of agreement were narrower in Group 1 (Figures 1, 2 and 3).

Conclusion In patients with hyperlactatemia, a global tissular perfusion marker, venous oxygen saturations presented a higher correlation and narrower bias and limits of agreement, suggesting, perhaps, that under high arterial lactate levels there is a generalized hypoperfusion that reflects not only on the $\mathrm{SvO}_{2}$, but also on the $\mathrm{SvcO}_{2}$. There was no agreement between those variables either.

References

1. Dellinger RP, Levy MM, Rhodes A, et al:. Surviving sepsis campaign: international guidelines for management of severe sepsis and septic shock: 2012. Crit Care Med 2013, 41:580-637.

2. Van Beest et al:: No agreement of mixed venous and central venous saturation in sepsis, independent of sepsis origin. Crit Care 2010, 14:R219.

3. Varpula M, Karlsson S, Ruokonen E, Pettilä V: Mixed venous oxygen saturation cannot be estimated by central venous oxygen saturation in septic shock. Intensive Care Med 2006, 32:1336-1343.

\section{Surgery/Trauma}

\section{P57}

Excessive fluid balance effect in mortality rate from surgical patients JM Silva Jr, FAM Nogueira, PMM Vianna, MCP Filho, CS Neucamp, VPL Maia, AMRR de Oliveira, MJC Carmona, LM Sá Malbouisson Hospital das Clinicas, Cerqueira Cesar, São Paulo, SP, Brazil Critical Care 2013, 17(Suppl 3):P57 (doi: 10.1186/cc12673)

Introduction In some studies including small populations of patients undergoing gastrointestinal surgery, a liberal infusion of fluids during the intraoperative period was associated with increased morbidity when compared with restrictive strategies for volume replacement. However, there are few studies evaluating the role of excessive fluid infusion in a population of high-risk surgical patients intraoperatively. The aim of this study was to evaluate the impact of the excessive water balance in the perioperative morbidity and mortality of high-risk surgical patients.

Methods A prospective cohort study during 1 year in four ICUs from three tertiary hospitals. The study included patients who required postoperative ICU, aged $\geq 18$ years undergoing major surgery. Patients with palliative surgery were excluded. The calculation from fluid balance was based on the surgical duration, the time of fasting preoperative and intraoperative, urine output and fluid replacement intraoperatively.

Results The study included 479 patients. Mean age was $61.2 \pm 17.0$ years and $51.1 \%$ were men. Nonsurvivors in the hospital represented $8.8 \%$ of patients. The median duration of surgery was 4.0 (3.2 to 5.5) hours. The average value of the SAPS 3 score was $41.8 \pm 14.5$ and ASA II was $52.8 \%$. However, when comparing survivors and nonsurvivors, the fluid balance intraoperatively from nonsurvivors was higher $(1,950(1,400$ to 3,400$) \mathrm{ml}$ vs. $1,400(1,000$ to 1,600$) \mathrm{ml}, P<0.001)$, patients with fluid balance above $2,000 \mathrm{ml}$ intraoperatively had longer ICU stay (4.0 (3.0 to 8.0 ) vs. 3.0 (2.0 to 6.0$), P=0.000$ ) and higher incidence of infectious complications $(41.9 \%$ vs. $25.9 \%, P=0.001)$, neurological $(46.2 \%$ vs. $13.2 \%, P=0.000)$, cardiovascular $(63.2 \%$ vs. $39.6 \%, P=0.000)$ and respiratory $(34.3 \%$ vs. $11.6 \%, P=0.000)$. In multivariate analysis, the fluid balance was an independent factor for death $(\mathrm{OR}=1.002, P=0.04$, $95 \% \mathrm{Cl}=1.001$ to 1.004$)$. See Table 1 .

Table 1 (abstract P57). Independent variables of hospital death

\begin{tabular}{lcccc}
\hline & & & \multicolumn{2}{c}{$95 \% \mathrm{Cl}$} \\
\cline { 5 - 6 } & P value & OR & Lower & Upper \\
\hline Males & 0.170 & 1.697 & 0.797 & 3.610 \\
SAPS 3 & 0.002 & 1.051 & 1.019 & 1.084 \\
ASA & 0.010 & 1.728 & 1.143 & 2.613 \\
Intraoperative blood transfusion & 0.645 & 1.210 & 0.539 & 2.715 \\
Fluid balance intraoperatively (ml) & 0.042 & 1.002 & 1.001 & 1.004 \\
Vasopressors intraoperatively & 0.328 & 1.531 & 0.652 & 3.596 \\
\hline
\end{tabular}

Variables included in the model: sex, SAPS 3, ASA, transfusion, fluid balance intraoperatively, intraoperative vasoactive drugs.

Conclusion Patients with excessive fluid balance intraoperatively have more ICU complications and higher hospital mortality.

P58

Implementation of an institutional protocol for rational use of blood products and its impact on postoperative cardiac surgery PGM de Barros e Silva, AC do Amaral Baruzzi, JT Garcia, MJ Rodrigues, MA Mieza, N Lasta, V Furlan, VA Fernandes

Hospital Totalcor, Cerqueira César, São Paulo, SP, Brazil

Critical Care 2013, 17(Suppl 3):P58 (doi: 10.1186/cc12674)

Introduction Cardiac surgeries are sometimes followed by significant blood loss and transfusions may be necessary. However, indiscriminate use of blood components can result in detrimental effects for the patient. In this study, we evaluated the short-term effects of the implementation of a protocol for the rational use of blood products in the postoperative period of cardiac surgery.

Methods Between April and June 2011 an institutional protocol was implemented in a private hospital specialized in cardiology to encourage rational use of blood products with the consent and collaboration of seven cardiac surgery teams. Clinical and demographic data of patients were collected, and the use of blood products and clinical outcomes during in-hospital period 6 months before and after implementation of the protocol were analyzed. The protocol consisted of an institutional campaign with educational intervention in the surgical, intensive care and anesthesiology teams aiming to spread the practice of blood transfusion based on clinical goals (anemia with hemodynamic changes, significant ventricular dysfunction), as well as making routine prescription of epsilon aminocaproic acid (EACA) intraoperatively. Comparisons between categorical variables were performed with the chi-square test and $P<0.05$ was considered statistically significant.

Results After 3 months of implementation of the protocol, the use of EACA rose from 31 to $100 \%$. The surgeries requiring any blood transfusion were $67 \%$ before the implementation of the protocol, and $40 \%$ in the subsequent months of the same year after implantation $(P<0.001)$. Clinical outcomes related to blood transfusion are presented in Table 1 separating both periods.

Table 1 (abstract P58)

\begin{tabular}{lcc}
\hline & $\begin{array}{c}\text { Group 1 } \\
\text { (pre-protocol) }\end{array}$ & $\begin{array}{c}\text { Group 2 } \\
\text { (after the protocol) }\end{array}$ \\
\hline Acute renal failure (\%) & 9 & 6 \\
Infection (\%) & 18 & 19 \\
Mortality (\%) & 3 & 3 \\
\hline
\end{tabular}

Conclusion The rational use of blood products associated with infusion of $\varepsilon$-aminocaproic acid has the potential to reduce the number of blood transfusions in postoperative of cardiac surgery, which can impact the risk of complications. 
P59

Role of hypothermia in the immediate postoperative period on mortality in a surgical ICU

AR Santana, FF Amorim, FB Soares, LG de Souza Godoy,

$\mathrm{L}$ de Jesus Almeida, TA Rodrigues, GM de Andrade Filho, TA Silva,

LC de Carvalho Santos, MPB de Araújo, PN Ferreira Jr, APP Amorim,

EB de Moura, JA de Araújo Neto, M de Oliveira Maia

Unidade de Terapia Intensiva Adulto do Hospital Santa Luzia, Asa Sul, Brasília,

DF, Brazil

Critical Care 2013, 17(Suppl 3):P59 (doi: 10.1186/cc12675)

Introduction Surgical patients are submitted to numerous factors that may cause postoperative hypothermia, such as a cool operating room environment, cold intravenous fluids and blood, cold antiseptic skin preparations and anesthetic-induced impairment of thermoregulatory control. Hypothermia may increase susceptibility to surgical wound infection, length of stay, intraoperative blood loss, morbid cardiac events, postoperative shivering, coagulopathy and also altered duration of drug action. The objective of this study was to evaluate the impact of hypothermia at ICU admission on hospital length of stay and mortality in a surgical ICU.

Methods A prospective cohort study conducted on patients admitted to the ICU of Hospital Santa Luzia, Brasilia, Brazil, during the period of 1 year. Hypothermia was defined as axillary temperature inferior to $35.5^{\circ} \mathrm{C}\left(95.9^{\circ} \mathrm{F}\right)$. Patients were divided into groups with hypothermia (HG) and without hypothermia (NG).

Results A total of 484 patients were enrolled. Mean age was $59 \pm 16$ years and $52.5 \%$ were male. Seventy-eight patients (16.1\%) were submitted to emergency surgeries. Mean APACHE II score was $8 \pm 5$, mean SAPS II was $16 \pm 10$. Twenty-four patients $(5 \%)$ had hypothermia at the time of ICU admission. The general mortality rate at 7 days, 28 days and hospital mortality was $0.8 \%(n=4), 1.9 \%(n=9)$ and $4.1 \%$ $(n=20)$, respectively. Patients with hypothermia had higher APACHE II score $(15 \pm 12$ vs. $8 \pm 4, P=0.00)$ and SAPS II $(25 \pm 17$ vs. $26 \pm 10$, $P=0.00$ ). There was no difference regarding the age of the groups and the hospital length of stay ( $4 \pm 6$ days in NG vs. $5 \pm 12$ days in HG, $P=0.76)$. However, the group with hypothermia had higher mortality rates $(20 \%$ vs. $4.3 \%, P=0.00)$. The relative risk for hospital mortality in patients with hypothermia at ICU admission was $4.6(95 \% \mathrm{Cl}: 1.02$ to 29.88).

Conclusion Few patients were hypothermic at the time of ICU admission in the immediate postoperative period. This may reflect the effectiveness of perioperative warming of the patients. Hypothermia at ICU admission was associated with greater severity scores and increased hospital mortality in this sample of surgical patients studied. References

1. Sajid MS, et al: Sao Paulo Med J 2009, 127:231-237.

2. Buggy DJ, Crossley AWA: Br J Anaesth 2000, 84:615-628.

3. Sessler DI: Anesthesiology 2001, 95:531-543.

Cite abstracts in this supplement using the relevant abstract number, e.g Santana AR, et al:: Role of hypothermia in the immediate postoperative period on mortality in a surgical ICU [abstract]. Critical Care 2013, 17(Suppl 3):P59. 